Abi Akle A., Yannou B., minel S. (2019). Information visualization for efficient knowledge discovery and informed decision in Design by Shopping. Journal of Engineering Design, 30 (6), 227-253, doi: 10.1080/09544828.2019.1623383.

\title{
Information visualization for efficient knowledge discovery and informed decision in Design by Shopping
}

\begin{abstract}
Design space exploration (DSE) describes the systematic activity of discovery and evaluation of the elements in a design space in order to identify optimal solutions by reducing the design space to an area of performance. Designers sample thousands of design points iteratively, explore the design space, gain knowledge about the problem and make design decision. The literature tells us that DSE results in a decision of quality called informed decision, which is supported by information visualization. The representation of design points is seen as primordial to gain an understanding of the problem and make an informed decision. In our work, we have sought to identify what type of graph is best suited to the discovery phase, and enables designers to make an informed decision. We designed a web platform with four design problems, and carried out an experiment with 42 participants. We found a graph that was better suited to making a decision of quality and to gaining greater understanding: the scatter plot matrix.
\end{abstract}

Keywords: visualization; computer aided design; decision making; design space exploration

\author{
Abbreviations \\ DbS: design by shopping \\ DSE: design space exploration \\ MCQ: multiple-choice questionnaire \\ PCP: parallel coordinate plot \\ SPM: scatter plot matrix \\ SSP: simple scatter plot
}

\section{Introduction}

There is a paradigm whereby designers shop for the best solution. Balling (1999) called this "design by shopping". Balling noted that the traditional optimization-based design process to "formulate the design problem, obtain analysis models and execute an optimization algorithm" leaves designers unsatisfied. Designers, like consumers, want to "shop" to gain an insight into trade-offs, and into feasible and impractical solutions, and learn about their alternatives before making decisions. Design by Shopping first enables designers to explore a design space, and then to optimize and choose a best solution from a set of possible designs, before finally developing realistic expectations with regard to what is possible. According to the classical design engineering process (Pahl and Beitz 2013), Design by Shopping is used during conceptual design and detailed design phases where analysis and synthesis reasoning are performed. More precisely, in Design by Shopping, designers apply these two stages of reasoning in an intertwined way with iterations until satisfactory design is achieved.

One embodiment of this paradigm is design space exploration (DSE) (Simpson et al. 2008). With DSE, designers sample thousands (and more) design points iteratively, explore the design space, which is a multidimensional set of data, gain insights and knowledge about the problem and make design decision. In DSE, the design decision is made following the discovery and evaluation of the elements in the design space in order to identify optimal solutions by reducing the design space to an area of performance. 
Abi Akle A., Yannou B., minel S. (2019). Information visualization for efficient knowledge discovery and informed decision in Design by Shopping. Journal of Engineering Design, 30 (6), 227-253, doi: 10.1080/09544828.2019.1623383.

Based on the work of Miller et al. (2013), exploring the design space comprises three main phases: (i) discovery: acquiring knowledge and understanding of the problem, (ii) narrowing: active pursuit of a design by eliminating sets, exploring limits, highlighting preferences, etc., and (iii) selection: checking satisfaction (see Figure 1). Based on the example provided by Stump et al. (2009), we identify that the discovery and narrowing phases are inseparable while the selection phase can be performed independently of the two others. Indeed, when designers reach this stage, they have the knowledge and understanding of the design problem and only Pareto optimal solutions remain in the design space. Thus, it is no longer necessary to narrow down into the design space. It is appropriate to compare performance solutions to identify those that are a good trade-off; and select a solution that adequately satisfies preferences. In addition, this phase can be performed by an additional person ("supra-decision-maker") i.e. disconnected from the first two phases when space is defined as optimal with a limited number of solutions that require inter and intra-criteria comparisons.

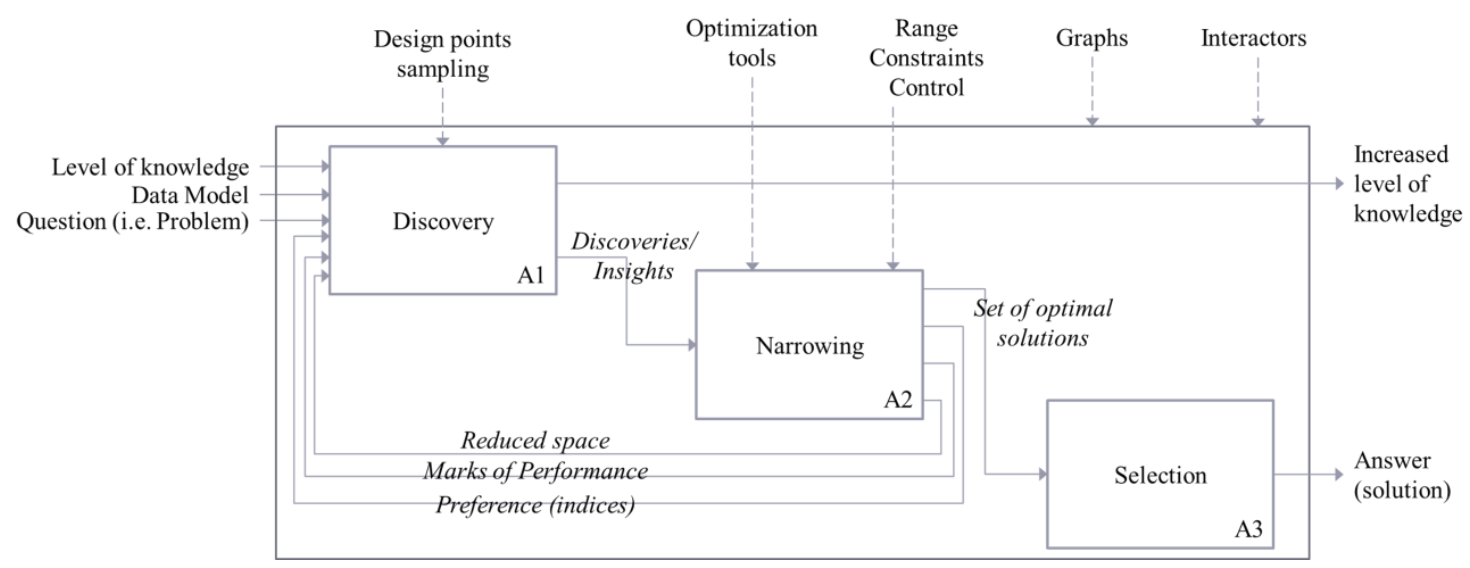

Figure 1. Design space exploration process

When addressing knowledge discovery in DSE, some authors refer to "informed decision making" (Sullivan et al. 2001; Mavris et al. 2010; Chandrasegaran et al. 2013). In fact, one key element of the Design by Shopping lies in the interaction between designers and possible solutions but more specifically the interaction with design parameters and performance variables. Indeed, the interaction impacts their understanding and/or preferences. In this way, the confidence which is gained for making decision is important. So, the impact of Design by Shopping process on the final result (i.e. the quality of picked solution) is as important as the process of better understanding which is the means. From our literature review on this decision type (see Section 2), we point to a need for information visualization for decision-makers.

Graphical supports are effective for design parameters (Simpson et al. 2007), engineering optimization (Barron et al. 2004) and conceptual design (Yannou et al. 2005). Graphs are also useful for quickly visualizing feasible solutions as opposed to impractical solutions, and those violating engineering constraints or client requirements. The data to be represented can be either: (i) the single vector of design parameters featuring the product solution, (ii) the single vector of solution performances for feasible solutions, and (iii) two sets of design parameters and corresponding performances for feasible and nonfeasible solutions (see for example the work of Canbaz (2013)). The already numerous studies in visual design have already shown that fast graphical design interfaces impact 
Abi Akle A., Yannou B., minel S. (2019). Information visualization for efficient knowledge discovery and informed decision in Design by Shopping. Journal of Engineering Design, 30 (6), 227-253, doi: 10.1080/09544828.2019.1623383.

user performance in terms of design efficiency, design effectiveness and the design search process (Ligetti et al. 2003).

We have identified several tools for exploring the design space. For example, the ARL Trade Space Visualizer (Stump et al. 2004), the VIDEO tool (Kollat, and Reed 2007), the LIVE tool (Yan et al. 2012) and the Rave tool (Daskilewicz and German 2012). We find various graphs in these tools: scatter plot matrix, 2D or 3D scatter plot, parallel coordinates plot, bar chart and tree map. In addition, it has already been shown in a simplified framework that the parallel coordinates plot is the most suitable graph for selection in Design by Shopping (Abi Akle, Minel, and Yannou 2015, 2017). This work focused on selecting a better design solution from among numerous alternatives in design by shopping. This experimental study identified a graph allowing designers to select one best solution with a high level of confidence in their decision. In our work, we are proposing to identify more precisely the implication of graphs for decision-making during design by shopping, and especially during the three phases of the process (Discovery, Narrowing and Selection) in a Multi-objective decision-making (MODM) situation when the design space is continuous with an infinity of possible solutions. It therefore appears that both the knowledge discovery phase for insight gain and understanding of the design problem are key elements in Design by Shopping or in other words "insights gain" (Zhang et al. 2012). We observe that information visualization is an indispensable element for the practice of DSE. Our research sought to answer the question: what graph best enables designers to be effective in the discovery phase and make an informed decision?

Our situation is a case-representation of multidimensional sets of data with an unlimited number of alternatives (design points). So, we were interested only by graphs allowing the visualization of multidimensional set of data. We thus identified three graphs useful for representing multidimensional sets of data ( $>3$ variables) and with an unlimited number of design points: simple scatter plot (SSP), scatter plot matrix (SPM) and parallel coordinate plot (PCP) (justification provided in section "Suitable Graphs"). We carried out experiments with 42 participants who were in final year of a MSc in Computer Aided Engineering and Product Development. In consequence, we considered them as novice designers. We designed a web platform with four design problems to solve for the novice designers. The platform lets us generate an unlimited number of design points (random or Pareto sampling), to shrink the design space with a range constraints controller, to visualize preferences, etc. in order to mimic the design activity.

\section{Informed decision}

The concept of "informed decision", met in several fields of enquiry, seems important for being ready and able to make a decision of quality.

In the field of monitoring and supervision, Ireson (2009) states that "the management of this mass of information is crucial in aiding the decision-making process, ensuring, as far as possible, that the responders have full situational awareness to make informed decisions". With the same idea, Bass (2000) and Riveiro, Falkman, and Ziemke (2008) indicate the need for "situational awareness" for the formulation of an informed decision. Bass adds the need for "fusing data into information and knowledge, so network operators can make informed decisions" (Bass 2000). In this field, it appears that "situational awareness" and the "transformation" of data into information and, then, in knowledge, contribute to the formulation of an informed decision. 
Abi Akle A., Yannou B., minel S. (2019). Information visualization for efficient knowledge discovery and informed decision in Design by Shopping. Journal of Engineering Design, 30 (6), 227-253, doi: 10.1080/09544828.2019.1623383.

In business and marketing, Lurie and Mason (2007) suggest that managing a large data set and the use of visualization tools could favour informed decision. Glaser and Tolman (2008) link informed decision to the process of analysing large amounts of data, "tracking" performance, and detecting patterns and trends. In this field, it appears that "manipulation and management" and "visualization" of large data sets and "knowledge and insight gains "by detecting patterns and trends contribute to the formulation of an informed decision.

The field of information systems tells us that "the making of informed decisions requires the application of a variety of knowledge to information" (Wiederhold 1992). In this field, it appears that the "transformation" of knowledge to information contributes to the formulation of an informed decision.

In the building field, making informed design decision requires managing a large amount of information on the detailed design options and properties, and running simulations of their performances: the designer needs a large design space and an overview of the consequences of parameter changes to gain a deep understanding of the performances, and so make an informed design decision (Petersen and Svendsen 2010). Russell, Chiu, and Korde (2009) consider that "visual analytics, the science of analytical reasoning facilitated by interactive visual interfaces, has the potential to improve the construction management process through the enhanced understanding of project status and reasons for it, better informed decision making". In this field, it appears that "manipulation and management" of a large amount of information, "analysis and treatment " by running simulations of performances, "knowledge and insight gains" to gain a deep understanding of the performance, the "understanding" of the consequences of parameter changes and "visualization", that is to say the visual analytics, contribute to the formulation of an informed decision.

In information visualization, Keim et al. (2006) indicate that for an informed decision, "it is indispensable to include humans in the data analysis process to combine flexibility, creativity, and background knowledge with the enormous storage capacity and the computational power of today's computers". Later, Keim et al. (2008a, 2008b) state that "visual analytics" is the system whereby an informed decision is made. It guarantees the full support of the user in navigating and analysing the data, memorizing insights and making informed decisions. We also read that "a tight coupling between cognition, interaction and visual analytics is necessary to enable the user to make informed decisions" (Meyer et al. 2010). In this field, it appears that including "human and cognition" in the "analysis and treatment" for "manipulation and management", and the use of "visualization" for "transformation" and so "knowledge and insight gains" contribute to the formulation of an informed decision.

Finally, we encounter informed decision in the field of engineering design. Wood, Otto, and Antonsson (1992) in preliminary design, model and manipulate uncertainties in a computer-assisted environment, under the hypothesis that doing so will enable the designer to make faster and better-informed decisions. Sullivan et al. (2001) showed, in particular, that to make informed decisions about the choice of design rules and clustering of design parameters, designers needed to know how changes in the environment would affect them. Chandrasegaran et al. (2013) indicate that "an effective computer support tool that helps the designer make better-informed decisions requires efficient knowledge 
Abi Akle A., Yannou B., minel S. (2019). Information visualization for efficient knowledge discovery and informed decision in Design by Shopping. Journal of Engineering Design, 30 (6), 227-253, doi: 10.1080/09544828.2019.1623383.

representation schemes". Mavris, Pinon, and Fullmer Jr (2010) argue that the integration of "visual analytics" in the design process provides designers with the ability to gain the knowledge and insights needed to make an informed decision. Visualization seems essential to facilitate the generation of hypotheses and the formulation of an informed decision. They point out that the data, knowledge, and insight necessary for the formulation of informed decisions are generated throughout the design process. In this field, it appears that the "manipulation and management" of uncertainties, the "visualization" with visual analytics, "analysis and treatment", "understanding" and "transformation" about the choice of design and hypothesis, "human and cognition" by focusing on the designers' choices and putting them in the simulation loop, and "knowledge and insight gain" contribute to the formulation of an informed decision.

From the literature, we collected 16 papers really contributing to this notion of informed decision. Consequently, we identified eight themes that appear to contribute to the formulation of an informed decision (see Table 1). We identified the four most widely used by authors, and which seem essential in the definition of an informed decision. These are: "knowledge and insight gain", "visualization", "analysis and treatment" and "manipulation and management".

Table 1. Themes that appear to contribute to the formulation of an informed decision

\begin{tabular}{|c|c|}
\hline Theme & Contributors \\
\hline $\begin{array}{l}\text { Situational } \\
\text { awareness }\end{array}$ & Ireson (2009), Bass (2000) \\
\hline $\begin{array}{l}\text { Knowledge and } \\
\text { insight gain }\end{array}$ & $\begin{array}{l}\text { Sullivan et al. (2001), Keim et al. (2006, 2008a, 2008b), Glaser } \\
\text { and Tolman (2008), Petersen and Svendsen (2010), Mavris, } \\
\text { Pinon, and Fullmer Jr (2010) }\end{array}$ \\
\hline Visualization & $\begin{array}{l}\text { Chandrasegaran et al. (2013), Lurie and Mason (2007), Bass } \\
\text { (2000), Mavris, Pinon, and Fullmer Jr (2010), Keim et al. (2006), } \\
\text { Russell, Chiu, and Korde (2009), Meyer et al. (2010), Riveiro, } \\
\text { Falkman, and Ziemke (2008) }\end{array}$ \\
\hline $\begin{array}{l}\text { Manipulation } \\
\text { and management }\end{array}$ & $\begin{array}{l}\text { Ireson (2009), Chandrasegaran et al. (2013), Lurie and Mason } \\
\text { (2007), Wood, Otto, and Antonsson (1992), Petersen and } \\
\text { Svendsen (2010), Mavris, Pinon, and Fullmer Jr (2010), Riveiro, } \\
\text { Falkman, and Ziemke (2008), Meyer et al. (2010) }\end{array}$ \\
\hline $\begin{array}{l}\text { Analysis } \\
\text { treatment }\end{array}$ & $\begin{array}{l}\text { Keim et al. (2006, 2008a, 2008b), Russell, Chiu, and Korde } \\
\text { (2009), Meyer et al. (2010), Riveiro, Falkman, and Ziemke (2008), } \\
\text { Mavris, Pinon, and Fullmer Jr (2010) }\end{array}$ \\
\hline Understanding & $\begin{array}{l}\text { Petersen and Svendsen (2010), Russell, Chiu, and Korde (2009), } \\
\text { Mavris, Pinon, and Fullmer Jr (2010) }\end{array}$ \\
\hline $\begin{array}{l}\text { Human } \\
\text { cognition }\end{array}$ & $\begin{array}{l}\text { Keim et al. (2006), Wood, Otto, and Antonsson (1992), Mavris, } \\
\text { Pinon, and Fullmer Jr (2010), Meyer et al. (2010) }\end{array}$ \\
\hline Transformation & $\begin{array}{l}\text { Wiederhold (1992), Chandrasegaran et al. (2013), Bass (2000), } \\
\text { Keim et al. (2006) }\end{array}$ \\
\hline
\end{tabular}

We can also match disciplinary fields to these elements. In Figure 2 we present the number of papers citing the key elements by field. We note that "situational awareness" is used exclusively by supervision and monitoring. If we focus on engineering design, dark grey in Figure 2, we distinguish four elements that seem to contribute most 
Abi Akle A., Yannou B., minel S. (2019). Information visualization for efficient knowledge discovery and informed decision in Design by Shopping. Journal of Engineering Design, 30 (6), 227-253, doi: 10.1080/09544828.2019.1623383.

widely to the formulation of an informed decision: "Knowledge and insight gain", "manipulation and management", "visualization" and "human and cognition".

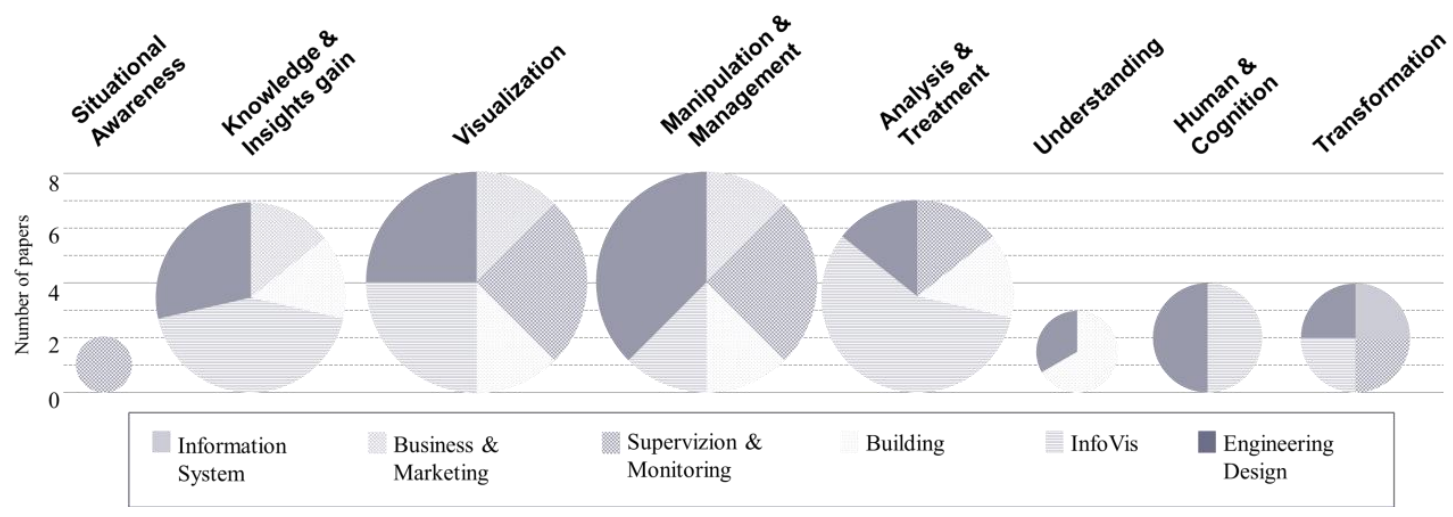

Figure 2. Number of papers indicating key elements per disciplinary field

We know that in engineering design, once a design has been formalized, a necessary design task is to make a selection from among candidate designs or parametric values (Otto and Antonsson 1993). The main challenge lies in resolving the inherent trade-offs that exist between the overall system and subsystems, and between conflicting and competing objectives (Abi Akle, Minel, and Yannou 2015, 2017). Thus, we define an informed decision in Design by Shopping as the selection of a design point, among several others, that will achieve optimal benefits and minimum inconvenience, following an iterative, interactive treatment and analysis process in which designers gain understanding, knowledge and insight with visualization and manipulation of large sets and / or data models.

\section{Suitable graphs}

In our context of design space exploration to make an informed decision, several graphs (design space representations) are available to us. The classification of Keim (2000) enabled us to determine what graphs were suited to the visualization of a multidimensional set of data. Indeed, Keim (2000) proposes to classify the information visualization according to three criteria: (i) interaction and distorsion technique, (ii) visualization technique and (iii) data to be visualized consisting of "one-dimensional", "two-dimensional", "multidimensional", "text web", "hierarchies graphs" and "algorithm/software". Our situation is a case-representation of multidimensional sets of data with an unlimited number of alternatives (design points). So, we were interested only by graphs allowing the visualization of multidimensional set of data: bar graph, simple scatter plot, scatter plot matrix, Chernoff's faces, star diagram, spider graph, value paths, parallel coordinates plot and combined or heatmap table.

Miettinen in her work (2014) provides a comparison of these graphs. Among all the comparison criteria she gives, two are interesting: number of criteria (i.e. design parameters) that can be visualized and the number of alternatives (i.e. design points) that can be visualized. In our case of design space exploration, we have a large number or unlimited number of design points to visualize. These recommendations allowed us to remove four graphs from our list of multidimensional graphs and therefore to keep: simple scatter plot, scatter plot matrix, parallel coordinates plot for the visualization of 
Abi Akle A., Yannou B., minel S. (2019). Information visualization for efficient knowledge discovery and informed decision in Design by Shopping. Journal of Engineering Design, 30 (6), 227-253, doi: 10.1080/09544828.2019.1623383.

unlimited number of design points and spider graph and combined or heatmap table for the visualization of large number of design points.

At last, we were interested by graphs best suited to multi-objective decision making (MODM) i.e. visualization of an unlimited number of alternatives. Moreover, we know that simple scatter plot and scatter plot matrix are best suited to the recognition of patterns (Wegman 1990), and parallel coordinates plot is the best for comparison and recognition of correlations (G. Andrienko and N. Andrienko 2001).

Thus, based on the work of Miettinen (2014), Wegman (1990), G. Andrienko and N. Andrienko (2001) and Keim (2000); we identified the simple scatter plot (SSP), the scatter plot matrix (SPM) and the parallel coordinates plot (PCP) (see Figure 3). We therefore set out to compare these three graphs for their ability to help making an informed decision in DSE.

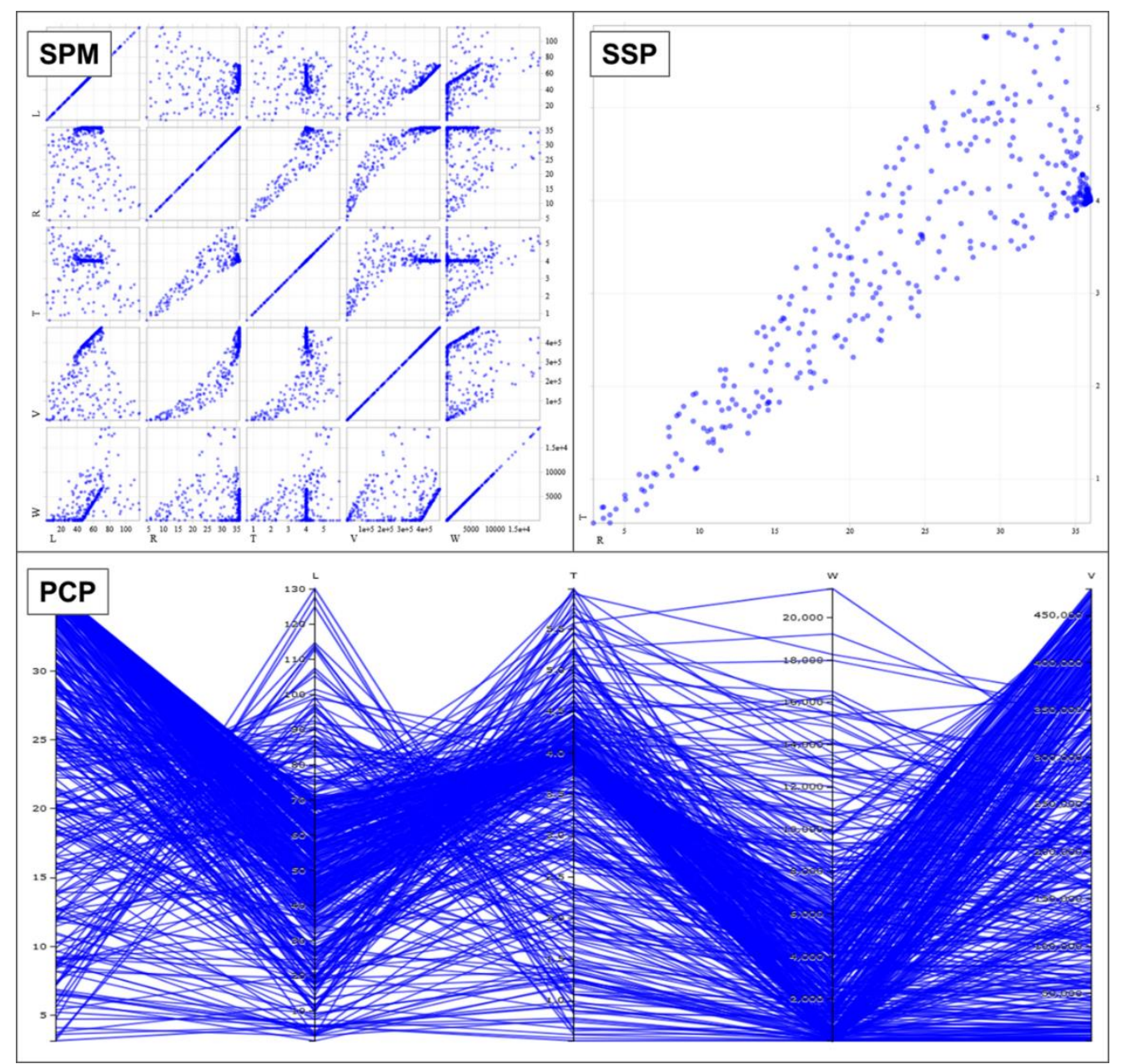

Figure 3. Illustration of the scatterplot matrix (SPM), the simple scatterplot (SSP) and the parallel coordinates plot (PCP)

A scatterplot is a conventional method to visualize the relationship between two variables. As a visual structure, the scatterplot uses position to encode the values of two variables and their relationships. This is a projection of the data (represented by the design points) in a 2D space. Siirtola (2007) considers that the scatterplot is useful for easily detecting non-linear patterns and positive or negative correlations between variables. Scatterplots are Cartesian representations, and so have a long history with, for example, 
Abi Akle A., Yannou B., minel S. (2019). Information visualization for efficient knowledge discovery and informed decision in Design by Shopping. Journal of Engineering Design, 30 (6), 227-253, doi: 10.1080/09544828.2019.1623383.

the learning of linear algebra at school. This early training results in a strong development of intuitions about the appearance of this type of representation (Wegman 1990).

The scatterplot matrix is a collection of simple scatterplots $(x-y)$ ordered in pairs. This representation provides an overview of data. The size of the matrix depends on the number of visualized variables. The scatterplots are duplicated in the matrix relative to the diagonal. It is possible to use colour glyphs, shape, or a size marker to add a supplementary variable. Ware (2004) points out that although colour used with scatterplots allows the identification of clusters and patterns, interpretation could be difficult.

A parallel coordinates plot is a graph displaying multiple criteria without drastically increasing the complexity of the display (Inselberg 2009). This graph was designed to work on high dimensional problems. It avoids the limits of orthogonal coordinate systems by placing each axis of coordinates in parallel. The design of a PCP is done in 4 steps:

- We start from a simple scatterplot with 3 design points (Figure 4.a.)

- We operate a projection of the points on the y-axis and on the $x$-axis (Figure 4.b.)

- Then we connect the coordinates of the points projected on the 2 axes by lines (Figure 4.c.)

- Finally, we place the axes in parallel (Figure 4.d.).

In a PCP, variable values are displayed on separate axes laid out in parallel. The design points (or alternatives) are depicted as profile lines that connect points on the respective axes.

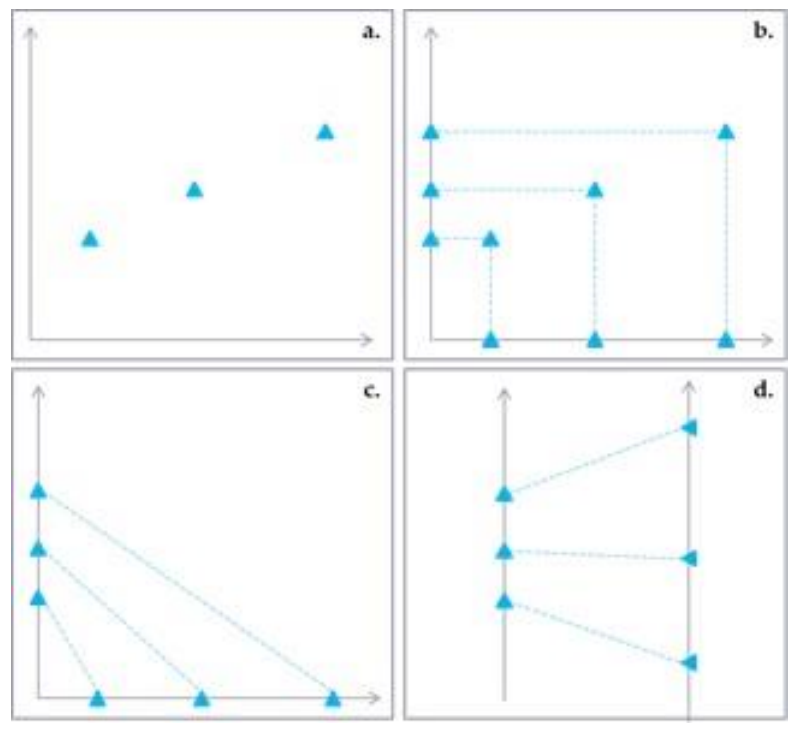

Figure 4. Design of Parallel Coordinates Plot

According to Gettinger et al. (2013), this representation can be readily interpreted and provides a good overview. Furthermore, patterns such as positive, negative and nontrivial (multiple) correlations, can quickly be identified at a glance.

\section{Experimental design}

To answer our research question "what graph best enables designers to be effective in the 
Abi Akle A., Yannou B., minel S. (2019). Information visualization for efficient knowledge discovery and informed decision in Design by Shopping. Journal of Engineering Design, 30 (6), 227-253, doi: 10.1080/09544828.2019.1623383.

discovery phase and make an informed decision?" we conducted a controlled experiment that adopted a between-subject approach. Each participant performed the experiment on one graph and solved four design optimization problems. They are classic problems of mechanical design from the literature: two-member Truss design (Truss), gear train design (Gear), multiple-disk clutch brake design (Disk) and pressure vessel design (Vessel). The first three problems come from the work of Deb and Srinivasan (2006) and the fourth from (Canbaz 2013).

All four design activities are of parametric sizing type. The designers, although in a parametric sizing activity, manipulate iteratively and or / simultaneously, the design parameters and the performance variables. Thus, they undertake "synthesis" reasoning by manipulating design parameters to reach performance and "analysis" by manipulating performance variables to identify design choices / preferences i.e. design parameters.

To conduct the experimentation we developed a web platform (http://these.aaa.alwaysdata.net/expe2/), where the problems and the three graphs are available. This platform allows, among other possibilities, the generation of design points (random or non-dominated Pareto solution sampling), glyph colouring according to designer preferences, and reduction of the design space.

\section{Procedure}

The experiment was divided into two main phases: (i) training, and (ii) testing. We also incorporated a milestone device into the session: multiple choice question forms. We used three forms, one at the beginning, one between training and testing, and one at the end of the session.

As already pointed out, we carried out experiments with 42 participants who were in final year of a MSc in Computer Aided Engineering and Product Development. In consequence, we considered them as novice designers. So, the experimentation was sized to fit into a two-hour session. The training part was used to upgrade the level of knowledge of participants. It was divided in three steps: a crash course, a "getting started" step with a tutorial to resolve the Truss problem, and a practice phase with the platform guide where participants solved the Gear problem. The test part was the phase when the graphs were tested and we performed our measurements. The tests were performed on two design problems consecutively without help of supports: the Vessel problem followed by the Disk problem. The instruction for both problems was to solve the biobjective optimization problem using the method of DSE to select an optimal solution with a justified means. The optimization problems are bi-objectives (see section Design problems), i.e. there are two antagonistic performance variables to maximize or minimize. For each of the two problems, the participants had a 10-minute time limit, and after each problem, a questionnaire was given (see section Measurements) to the participant to raise confidence, and assess the information acquired during exploration, which enabled the participant to make their decision (selecting a solution). The sequence of parts and steps in the experiment is illustrated in Figure 5. 
Abi Akle A., Yannou B., minel S. (2019). Information visualization for efficient knowledge discovery and informed decision in Design by Shopping. Journal of Engineering Design, 30 (6), 227-253, doi: 10.1080/09544828.2019.1623383.

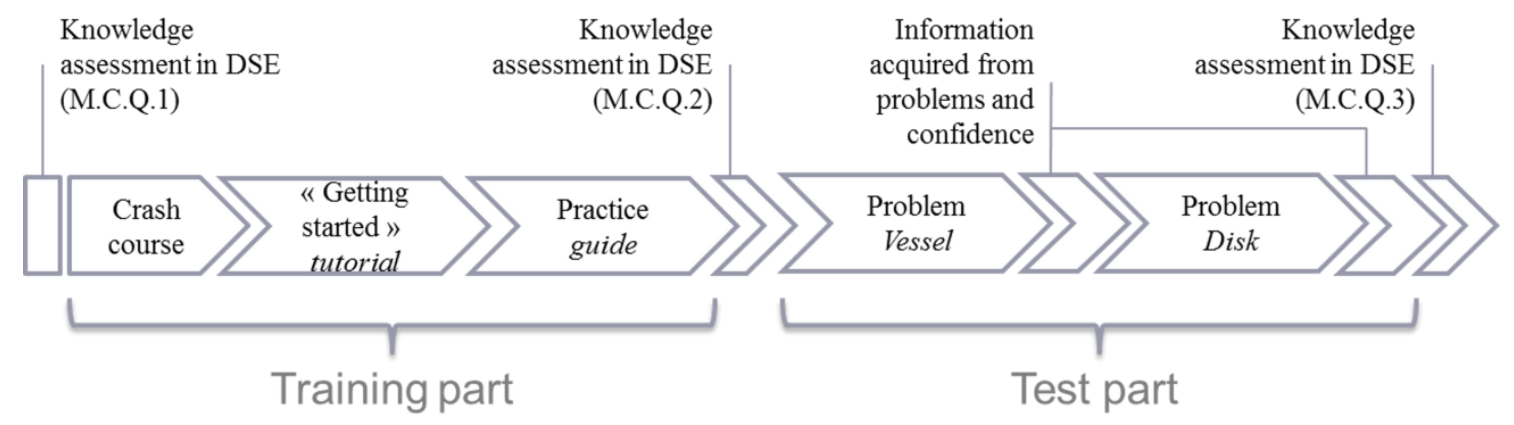

Figure 5. Sequence of the experiment

\section{Design of multiple-choice questionnaires}

In our experiment, we have designed three multiple-choice questionnaires (MCQs) each one with specific function. The first one was used to define the designer profile. While, the second and third MCQs were used to control knowledge evolution of the participants. Each MCQ consisted of ten questions (each with three possible answers) related to the exploration and visualization of design space. As each group of participants performed the experiment on one type of graph and we needed three MCQs for each experiment session, we designed nine MCQs which corresponded to three MCQs for each type of graph. Thus, questions about visualization were contextualized to the type of graph tested. However, these questions are similar to avoid bias. Indeed, we did not want a group of participants to be favoured by easier questions.

For example, question \#8 of MCQ \#1 is shown in Figure 6. The question is the same but the illustration is based on the type of graph tested. In addition, the MCQ \# 1 for the SPM group is given in appendices.

What is the interaction between variables $A$ and $B$ ?
a) Clusters
b) 2 transition points
c) A correlation
d) I do not know

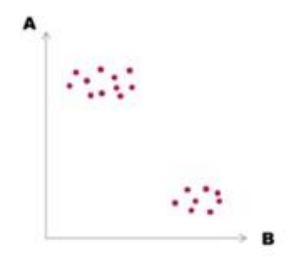

What is the interaction between variables $A$ and $B$ ?
a) Clusters
b) 2 transition points
c) A correlation
d) I do not know

Figure 6. Example of question \#8 from MCQ \#1

In the experiment, each of the three MCQs had a specific function. The first MCQ was done before starting the experiment session and allowed us to know the «designer» 
Abi Akle A., Yannou B., minel S. (2019). Information visualization for efficient knowledge discovery and informed decision in Design by Shopping. Journal of Engineering Design, 30 (6), 227-253, doi: 10.1080/09544828.2019.1623383.

profile of the participant from his/her level of knowledge in exploration and visualization of design space. Thus, we defined three profiles by counting the number of correct answers to MCQ \#1:

- "Expert" for a score between 8 and 10 .

- "Intermediate" for a score between 4 and 7

- "Novice" for a score between 0 and 3

The second MCQ allowed us to control the evolution of knowledge of the participant and to check that $\mathrm{s} / \mathrm{he}$ reached at least an Intermediate level before going to the Test part.

The third MCQ allowed us to verify that the graph used during the Test part did not create confusion for the participant. We have verified that the number of correct answers between MCQ \#2 and MCQ \#3 did not decrease.

Finally, the three MCQs was for us the means to verify that there were no differences between the three groups of participants or more widely ensure us to perform statistical analysis with comparable groups. Indeed, we had three groups of participants:

- Participants performing the experiment with the SPM graph ( $\mathrm{n}=14$ subjects)

- Participants performing the experiment with the SSP graph ( $\mathrm{n}=14$ subjects)

- Participants performing the experiment with the PCP graph ( $\mathrm{n}=14$ subjects)

In addition, we potentially had participants with different levels of expertise: Expert, Intermediate and Novice. Figure 7 illustrates the different possible evolutions of knowledge in exploration and visualization of design space according to the designer profile.

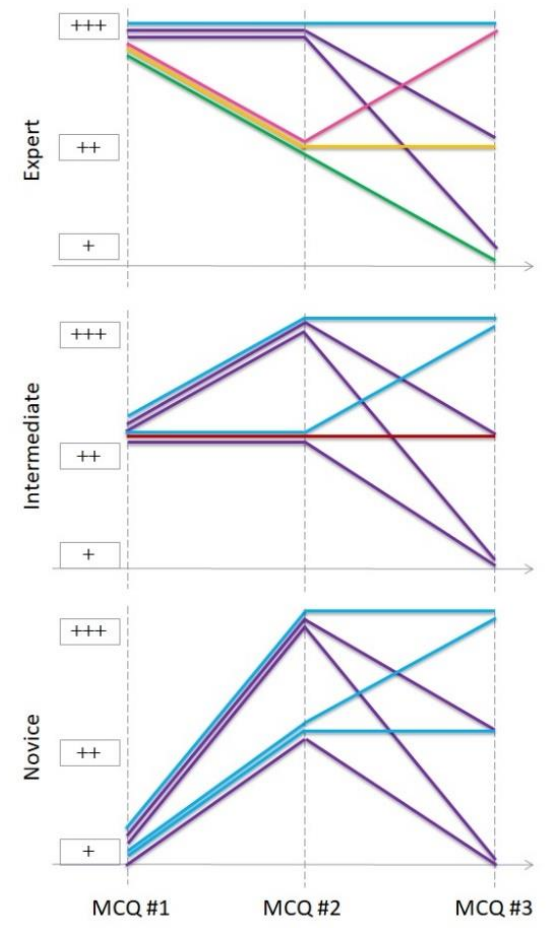

Figure 7. Illustration of the different "evolutions of knowledge" function of the designer profile 
Abi Akle A., Yannou B., minel S. (2019). Information visualization for efficient knowledge discovery and informed decision in Design by Shopping. Journal of Engineering Design, 30 (6), 227-253, doi: 10.1080/09544828.2019.1623383.

Blue lines represent the expected evolutions and in purple the cases where the test part creates confusion. We note three possible particular cases: (i) the constant intermediate in red, (ii) the experimentation creating a total confusion for the expert participant in green and (ii) the experts disturbed by the training part in pink and yellow.

\section{Experimental platform}

To perform the experimentation, we developed a platform available on the web at the URL: http://these.aaa.alwaysdata.net/expe2/. The interface of the platform is composed of four main zones (Figure 8). There is a first zone "Details about one design point" (see Box No. 1 in Figure 8) where the detail of a design point is displayed when the "selection" function is used, e.g. overflight of a design point in a graph highlights the parameter values of the design point. A second zone "Menu with functions" (see Box No. 2 in Figure 8) has the different tabs and features of the platform. There is another zone called "Info about design space" (see Box No. 3 in Figure 8) where there are indications such as a colour scale and the number of design points projected in the graph. Lastly, the zone "Visualization of the design space" (see Box No. 4 in Figure 8) displays the projection of the design points.

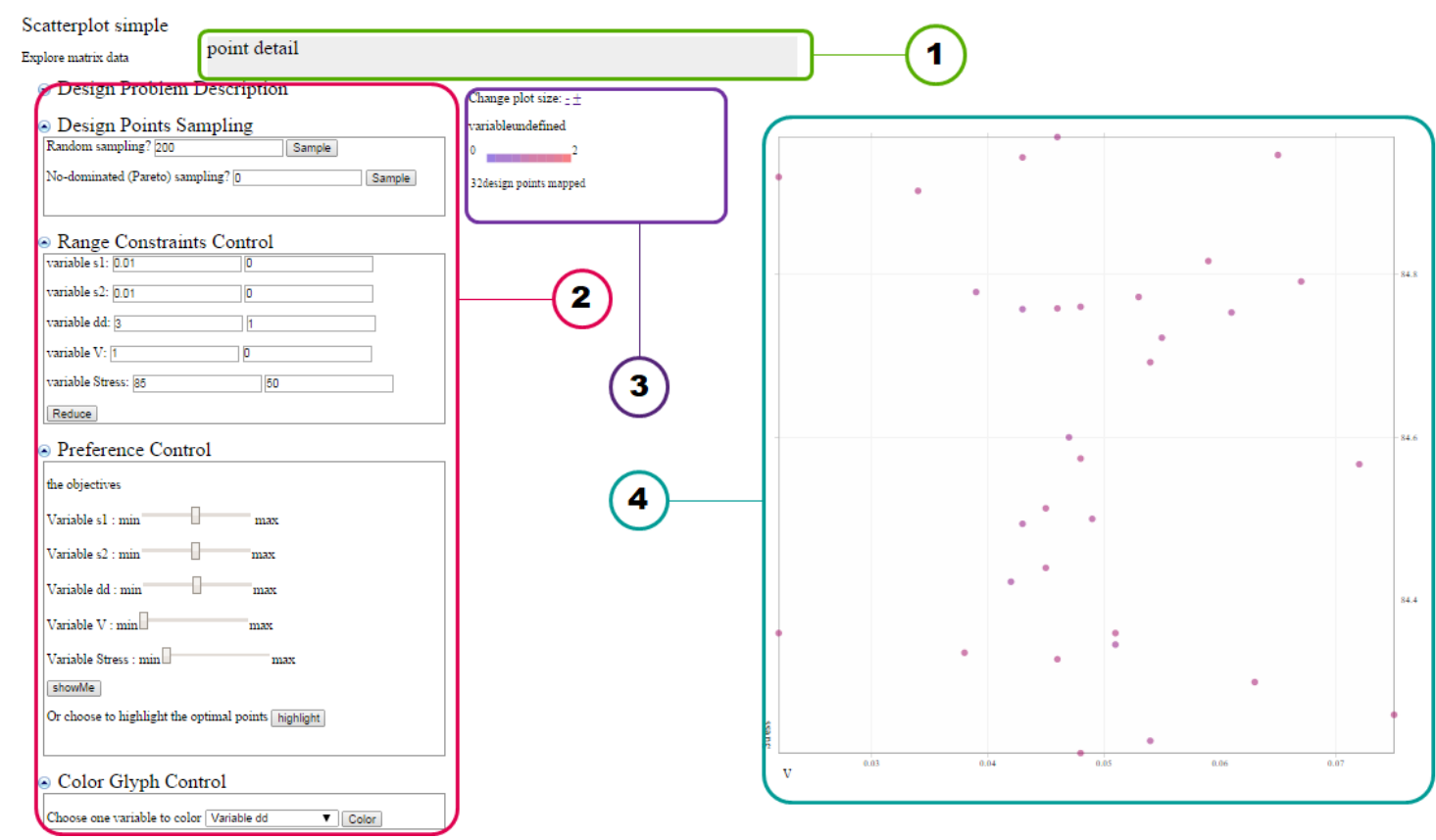

Figure 8. Screenshot of the platform interface with the four main zones. (1) Details about one design point; (2) Menu with functions; (3) Info about design space and (4) Visualization of the design space.

Concerning the functionalities available in the menu, we designed five major ones, plus two others in order to adapt interaction with graphs. The major functionalities are:

(1) Design problem description: this proposes the statement of a design problem. In this description, we find a diagram representing the problem, the presentation of the design variables, the performance variables and the objectives and constraints. 
Abi Akle A., Yannou B., minel S. (2019). Information visualization for efficient knowledge discovery and informed decision in Design by Shopping. Journal of Engineering Design, 30 (6), 227-253, doi: 10.1080/09544828.2019.1623383.

(2) Design points sampling: this allows the generation of feasible design points in the design space. Two point generators are available: "random sampling" produces the desired number of points in the design space randomly, and "non-dominated (Pareto) sampling" produces "non-dominated" points in the Pareto sense.

(3) Range constraints control: this allows the design space to be shrunk by controlling the max and min values of each variable.

(4) Preference control: this makes it possible to highlight the design points according to two "preference" options. The first option enables coloring of the design points on a colour scale in accordance with the objectives of the problem. The second option makes it possible to highlight the solutions (points) called optimal (in the sense of Pareto).

(5) Colour glyph control: this allows addition of a colour marker to a variable of choice.

\section{Design problems}

As stated above, we used four bi-objective design problems for our experiment. The first two were used during the training part; a description is available in (Deb and Srinivasan 2006). The first problem for the test was the Vessel problem, described as follows: it is a design problem of a cylindrical thin walled pressure vessel with hemispherical ends. There are three design variables $(R, T, L)$ and two performance parameters $(W, V)$. The objectives are minimizing $W$ and maximizing $V$ by controlling $R, T$ and $L$ while satisfying constraints $C_{1-7}$. The vessel problem nomenclature and constants are: $W$ the weight of the pressure vessel in (lb), $V$ the volume of the pressure vessel, $R$ the radius, $T$ the thickness of the pressure wall, $L$ the length of the cylinder, $P$ the pressure inside the cylinder, UTS the ultimate tensile strength of the vessel material, equal to $35 \mathrm{klb}, d$ the density of the vessel material, equal to 0.283, and Circ the circumferential stress (see Figure 9).

The second problem for the test was the Disk problem described as follows: a multiple clutch brake needs to be designed. Two conflicting objectives are considered: minimization of mass $(M)$ of the brake system in $\mathrm{kg}$, and minimization of stopping time $(S)$ in seconds. There are five decision variables $R_{\mathrm{i}}, R_{\mathrm{o}}, t, F$, and $Z$ (see Figure 9), where $R_{\mathrm{i}}$ is the inner radius in $\mathrm{mm}, R_{\mathrm{o}}$ is the outer radius in $\mathrm{mm}, t$ is the thickness of the disks in $\mathrm{mm}, F$ is the actuating force in $\mathrm{N}$, and $Z$ is the number of disks (or friction surfaces). All five variables are considered discrete, and their allowable value ranges are: $60<R_{\mathrm{i}}<80$, $90<R_{\mathrm{o}}<110,1<t<3,600<F<1000,2<Z<10$. All performance formulas, constraints and bounds of the two problems are available on the web platform. 


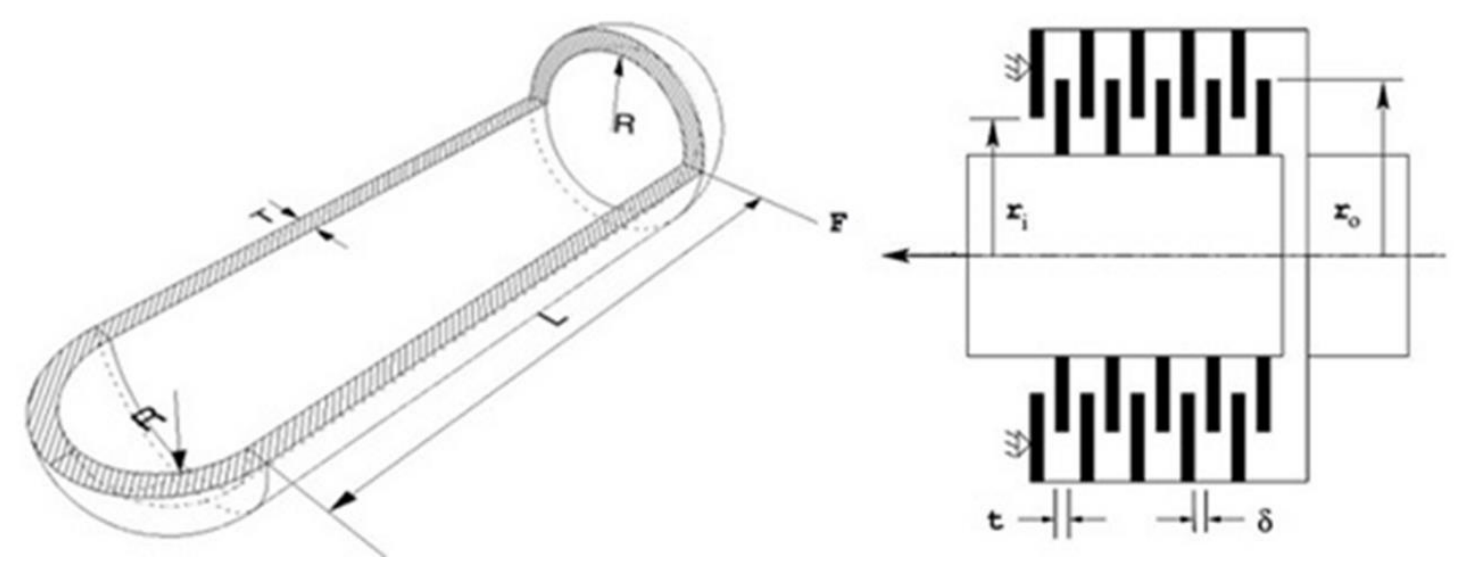

Figure 9. Illustration of the Vessel problem (Canbaz 2013) (left) and the Disk problem (Deb and Srinivasan 2006) (right)

\section{Measurements}

In our work, variables were either measured during the test with an eye-tracking system (Tobii X2) or collected through the questionnaires. We used three types of measurements: those controlled during the test and those collected with questionnaires. The controlled measurements were used to make sure there were no differences between the three groups of participants (one group per graph) concerning the knowledge level from the three multiple-choice questionnaires (MCQs) and the "performance" of the final solution selected (i.e. "is it a Pareto solution?").

The dominance is defined as: $y$ dominates $z$ if and only if $\forall i \in[1 \ldots n], f_{i}(y) \leq f_{i}(z)$ and $\exists k \in[1 \ldots n] \mid f_{k}(y)<f_{k}(z)$.

Dominance in the Pareto sense is defined as: One solution $x^{p} \in X$ is Pareto-optimal if and only if $\nexists x \in X$ such that $x$ dominates $x^{p}$, and where: we consider a multi-objective problem: $\min \left(F(x)=\left(f_{1}(x), f_{2}(x), \ldots, f_{n}(x)\right), n \geq 2\right.$

- $x \in X$

- $X$ is the decision space

- $Y$ is the objective space (or performance space)

- And $Y=F(X)$

We then have three measurements made during the test:

- The number of discoveries / insights gained by the participant, knowing that for each problem, there are seven discoveries in order to make a fully informed decision (see Table 2). The number of discoveries was collected by analysis of the video screen in conjunction with an eye-tracking record. Eye-tracking record gave us the eyes gaze on the screen step by step with seven focus points. For example, in Figure 10, we can observe a discovery made by participant $\mathrm{n}^{\circ} 11$ while resolving Disk problem. The eyes gaze, here, is depicted by the yellow connected circles. We detect in this example that the participant checks the name of the variable in the yellow circles \#1 and \#2 (under the \#7), 
Abi Akle A., Yannou B., minel S. (2019). Information visualization for efficient knowledge discovery and informed decision in Design by Shopping. Journal of Engineering Design, 30 (6), 227-253, doi: 10.1080/09544828.2019.1623383.

then s/he visualizes an interaction between variables, here a negative correlation, in the yellow circles \#3, \#4 and \#5. Finally, s/he checks, once again, the name of the observed variables in the yellow circles \#6 and \#7. Following the eyes gaze on all video recording, we can know if participants made a discovery. These records were analysed in combination with a post-problem questionnaire, in order to be sure thatthe discovery was made. The entire video recording with eye tracking recording of participant \#11 solving Disk problem is provided at this URL: https://www.youtube.com/watch?v=0-A6c57Jleg.

- We also measured the time elapsed before the participant made the first discovery (in seconds). This time was not dependent on the time required to understand the problem, because the timer was triggered only when the participant had read the description of the problem and made the first sampling of design points. This second measurement gave us a first clue to the speed of discovery with the three graphs.

- The third measurement was the average time to complete a discovery (in seconds), i.e. the total time to make all the discoveries divided by the number of discoveries made. This measurement gave us a second clue to the speed of discovery with the three graphs.

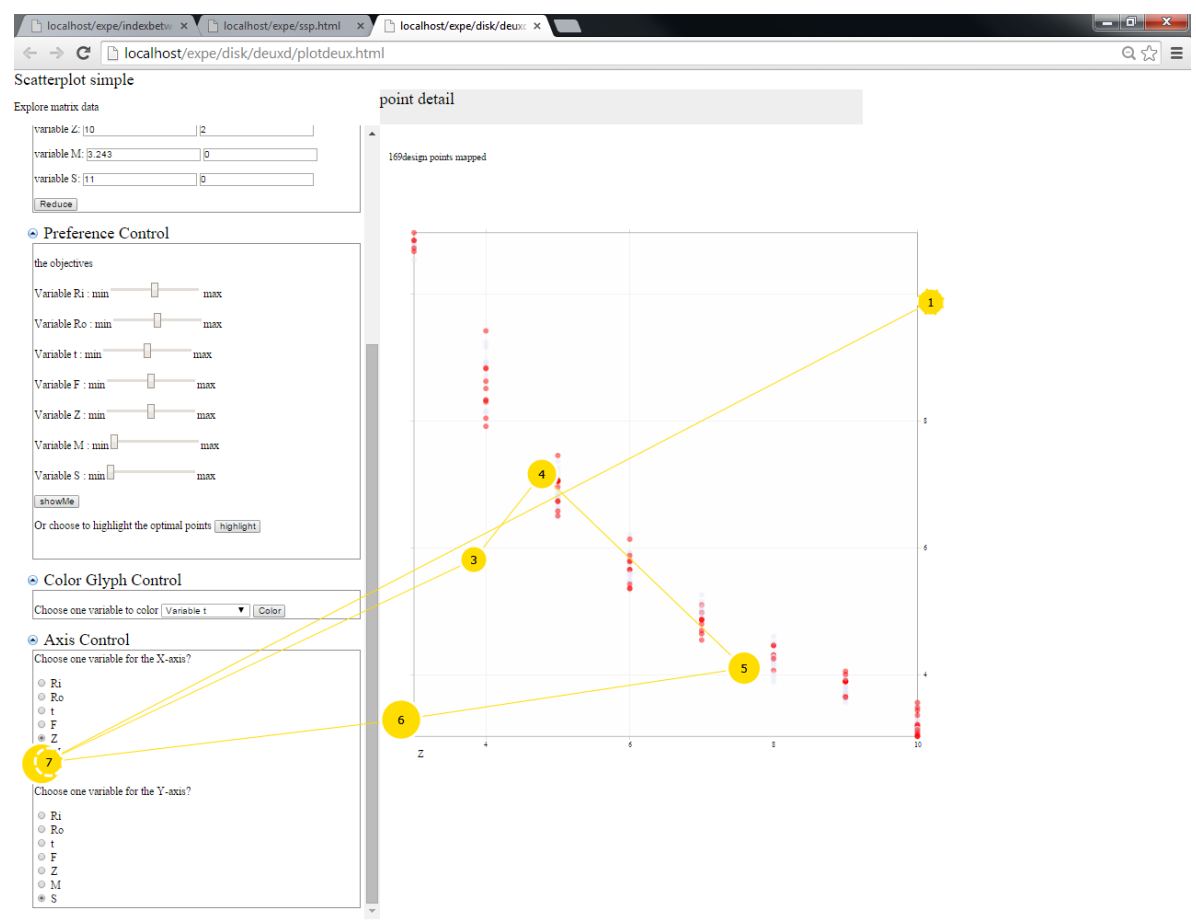

Figure 10. Screenshot of the Disk problem solving by participant \#11 combined with eyes gaze. 
Abi Akle A., Yannou B., minel S. (2019). Information visualization for efficient knowledge discovery and informed decision in Design by Shopping. Journal of Engineering Design, 30 (6), 227-253, doi: 10.1080/09544828.2019.1623383.

Table 2. List of the discoveries for the two problems of the test

\begin{tabular}{|c|c|c|}
\hline Problem & $\begin{array}{l}\text { Discoveries from a global point of } \\
\text { view }\end{array}$ & $\begin{array}{l}\text { Discoveries from a local point of } \\
\text { view (Pareto solutions) }\end{array}$ \\
\hline Vessel & $\begin{array}{l}\text { Positive correlation (trend) between } \\
R \& V \\
\text { Positive correlation (trend) between } \\
R \& T \\
\text { Positive correlation (trend) between } \\
T \& V\end{array}$ & $\begin{array}{l}\text { Positive correlation between } W \& L \\
\text { Positive correlation between } V \& L \\
\text { The solutions tend to } T=4 \\
\text { The solutions tend to } R=36\end{array}$ \\
\hline Disk & $\begin{array}{l}\text { Negative correlation } \\
\text { between } S \& Z\end{array}$ & $\begin{array}{l}\text { Positive correlation (trend) between } \\
R_{\mathrm{i}} \& R_{\mathrm{O}} \\
\text { A transition point for } R_{\mathrm{O}}=100 \\
\text { A transition point for } M=1 \\
\text { The solutions tend to } t=1 \\
\text { The solutions tend to } F=1000 \\
\text { Positive correlation between } M \& Z\end{array}$ \\
\hline
\end{tabular}

Finally, we used a post-problem questionnaire to determine whether participants could "justify" their decisions based on the information acquired during exploration (correlation, trend, transition point, etc.). In the analysis of this indicator, we determined only whether or not the participants justified their decision (nominal qualitative variable). We could not analyse all the information that the participants used to justify their response, because this amount depended on the participant. Similarly, blank forms were not considered (no response did not mean that participants did not know how to justify their decision).

\section{Results}

We had a sample of 42 subjects and used a between-group approach (three groups), so we had three samples of $N=14$ subjects in each group / graph. For the analysis of data, we applied different statistical tests, and we considered a significance level $\alpha=10 \%$. The statistical tests chosen to perform the analyses are described in (Kanji 2006). We considered the following statistical hypotheses: H0: there is no difference between the use of graphs for the discovery phase and H1: there is a difference between the three graphs.

\section{Differences and similarities between the three groups of participants}

First of all, we analysed the data obtained with MCQs to verify whether we could distinguish designer profiles within testers and / or whether there was a difference between groups (i.e. three groups - three graphs).

To detect designer profiles, we use the Friedman test (and the Wilcoxon Signed-Rank test if a post hoc analysis was required), because MCQs answers gave us qualitative ordinal variables, we had three measures to compare (i.e. the three MCQs), and the groups were paired (i.e. within-group approach).

First, we wanted to know for each group if the scores obtained with the three MCQs were different. Thus, we tried to reject the statistical null hypothesis H0: There is no difference between the scores obtained with the three MCQs. 
Abi Akle A., Yannou B., minel S. (2019). Information visualization for efficient knowledge discovery and informed decision in Design by Shopping. Journal of Engineering Design, 30 (6), 227-253, doi: 10.1080/09544828.2019.1623383.

We sought to verify this result for each group of participants. We formulated the alternative hypothesis $\mathrm{H} 1$ for the group that tested the SSP: MCQ1(SSP)<>MCQ2(SSP) $<>M C Q 3(S S P)$; for the group that tested PCP: MCQ1(PCP ) $<>M C Q 2(\mathrm{PCP})<>\mathrm{MCQ} 3$ (PCP); and for the group that tested the SPM: MCQ1(SPM ) $<>$ MCQ2(SPM ) $<>M C Q 3(S P M)$.

Table 3 sums up the statistical results obtained, and the first line of the table gives the results of Friedman tests.

The p-values indicated that the results were significant. That is, there was a significant difference between the scores obtained at the three MCQs for the groups that tested the SSP, there was a significant difference between the scores obtained at the three MCQs for the groups that tested the PCP, and there was a significant difference between the scores obtained at the three MCQs for the groups that tested the SPM.

Based on these results, we performed Wilcoxon Signed-Rank tests because a posthoc analysis was required.

For the SSP group, rank averages were 1 for MCQ1, 2.2 for MCQ2 and 2.8 for MCQ3. Wilcoxon's tests gave us significant differences between MCQ1(SSP) and MCQ2(SSP) with $\mathrm{p}=0.0005$, and MCQ2(SSP) and MCQ3(SSP) with $\mathrm{p}=0.0041$. So, we had: MCQ1(SSP)<MCQ2(SSP)<MCQ3(SSP). SSP participants had higher scores at MCQs as the experiment progressed.

For the PCP group, rank averages were 1 for MCQ1, 2.1 for MCQ2 and 2.9 for MCQ3. Wilcoxon's tests gave us significant differences between MCQ1(PCP) and MCQ2(PCP) with $\mathrm{p}=0.0005$, and MCQ2(PCP) and MCQ3(PCP) with $\mathrm{p}=0.0006$. So, we had: MCQ1 $(\mathrm{PCP})<\mathrm{MCQ} 2(\mathrm{PCP})<\mathrm{MCQ} 3(\mathrm{PCP})$. PCP participants had higher scores on MCQs as the experiment progressed.

For the SPM group, rank averages were 1 for MCQ1, 2.2 for MCQ2 and 2.8 for MCQ3. Wilcoxon's tests gave us significant differences between MCQ1(SPM) and MCQ2(SPM) with $\mathrm{p}=0.0004$, and MCQ2(SPM) and MCQ3(SPM) with $\mathrm{p}=0.0336$. So, we had: MCQ1 $(\mathrm{SPM})<\mathrm{MCQ} 2(\mathrm{SPM})<\mathrm{MCQ} 3(\mathrm{SPM})$. SPM participants had higher scores on MCQs as the experiment progressed.

Table 3. Results of the Friedman and Wilcoxon tests for the intra-graph analysis

\begin{tabular}{|c|c|c|c|c|c|c|}
\hline \multicolumn{3}{|c|}{ Friedman test } & \multirow{2}{*}{$\begin{array}{l}\text { SSP } \\
\operatorname{csqr}=23.89, \mathrm{df}=2 \\
\text { and } p<0.0001\end{array}$} & \multirow{2}{*}{$\begin{array}{l}\text { PCP } \\
\operatorname{csqr}=26.14, \\
\text { df }=2 \\
p<0.0001 \\
\end{array}$} & & \multirow{2}{*}{$\begin{array}{l}\text { SPM } \\
\operatorname{csqr}=21.09, \mathrm{df}=2 \\
\text { and } p<0.0001\end{array}$} \\
\hline Friedman & & & & & and & \\
\hline \multirow[t]{2}{*}{$\begin{array}{l}\text { Wilcoxon } \\
\text { Signed } \\
\text { Rank Test }\end{array}$} & $\begin{array}{l}\text { MCQ1 } \\
\text { MCQ2 }\end{array}$ & vs. & $\begin{array}{l}W=-105 \\
Z=-3.28 \\
p=0.0005\end{array}$ & $\begin{array}{l}W=-105 \\
Z=-3.28 \\
p=0.0005\end{array}$ & and & $\begin{array}{l}W=-107.5 \\
Z=-3.36 \\
p=0.0004\end{array}$ \\
\hline & $\begin{array}{l}\text { MCQ2 } \\
\text { MCQ3 }\end{array}$ & vs. & $\begin{array}{l}W=-76, Z=-2.64 \\
\text { and } p=0.0041\end{array}$ & $\begin{array}{l}W=-103 \\
Z=-3.22 \\
p=0.0006\end{array}$ & and & $\begin{array}{l}W=-53, Z=-1.83 \\
\text { and } p=0.0336\end{array}$ \\
\hline
\end{tabular}

The results for the three graphs, presented in Table 3, led us to conclude that in each group all the participants had a novice profile at the start of the test, and all gained knowledge (in DSE): MCQ1 < MCQ2 < MCQ3. The average scores of the MCQs are given in Figure 11. 
Abi Akle A., Yannou B., minel S. (2019). Information visualization for efficient knowledge discovery and informed decision in Design by Shopping. Journal of Engineering Design, 30 (6), 227-253, doi: 10.1080/09544828.2019.1623383.

It was now necessary to check that there was no difference in scores between the groups. That is, we wanted to show that MCQ1(SSP) $\equiv$ MCQ1(PCP) $\equiv$ MCQ1(SPM); $\mathrm{MCQ} 2(\mathrm{SSP}) \equiv \mathrm{MCQ} 2(\mathrm{PCP}) \equiv \mathrm{MCQ} 2(\mathrm{SPM})$ and $\mathrm{MCQ} 3(\mathrm{SSP}) \equiv \mathrm{MCQ} 3(\mathrm{PCP}) \equiv$ MCQ3(SPM). To detect differences between groups, we used the Kruskal-Wallis test, because in this case the analysis was a between-group approach.

Table 4. Results of Kruskal-Wallis tests for the inter-graphs analysis

\begin{tabular}{lllll}
\hline & MCQ1 & MCQ2 & MCQ3 \\
\hline \multirow{2}{*}{ Kruskal-Wallis test } & $H=0.61 p=0.7371$ & $H=1.55 p=0.4607$ & $\begin{array}{l}H=4.2 \\
p=0.12\end{array}$ \\
\cline { 2 - 5 } Rank & SPM & 23.1 & 22 & 16.8 \\
\cline { 2 - 5 } & PCP & 19.5 & 18.4 & 26.3 \\
\cline { 2 - 5 } & SSP & 21.9 & 24.1 & 21.5 \\
\hline
\end{tabular}

The results of Kruskal-Wallis tests (see Table 4) and presented in Figure 11 show us that there was no significant difference between the three graphs for the three MCQs answers because the p-values obtained for the three tests were above $10 \%$. There was thus no difference in knowledge between the groups.

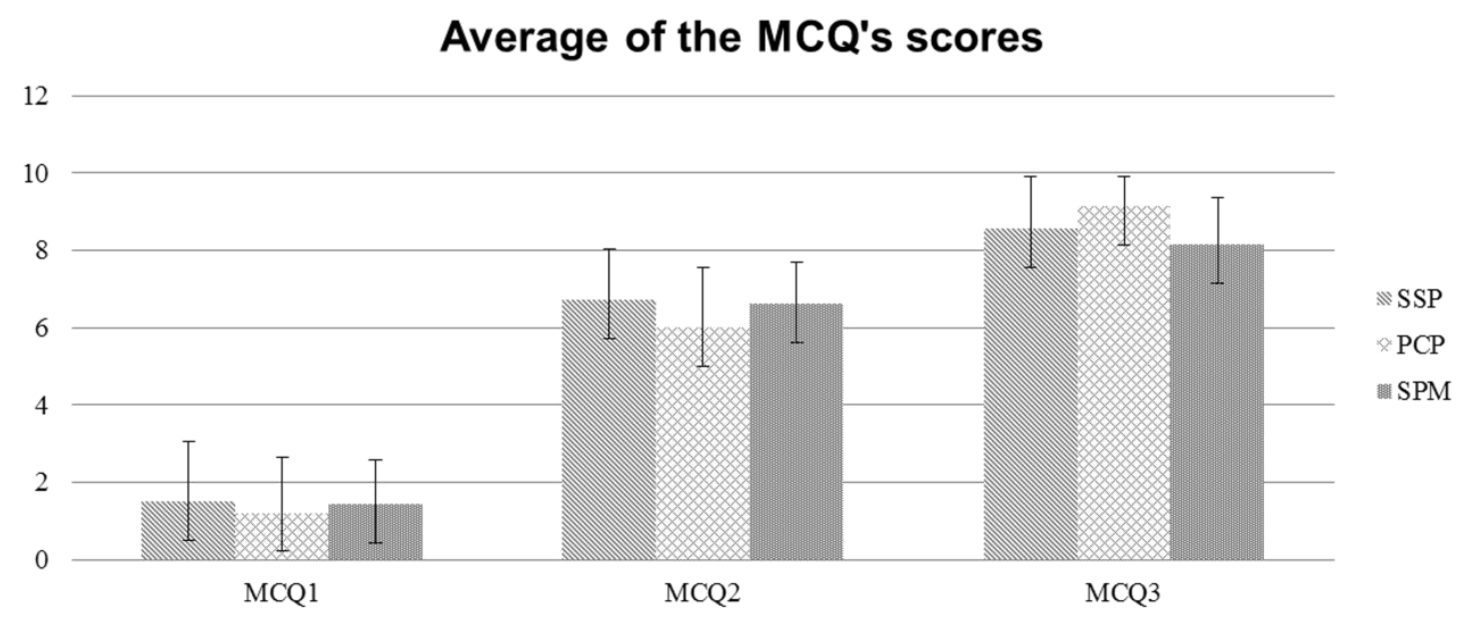

Figure 11. Average scores of MCQs and standard deviation

To conclude this part of statistical analysis, the level of knowledge in Design Space Exploration had evolved (between MCQ1, MCQ2 and MCQ3) for participants of the three groups (i.e. the three graphs). Moreover, there were no differences in responses to the three MCQs between the three groups i.e. the three groups had evolved in the same way and were therefore comparable for the following analyses.

We went on to analyse the solutions selected by the participants. For this indicator we analysed the data from the two problems together. To compare the type of solutions selected between the three groups (SPM, PCP, SSP) we used the Chi-square test because we have three groups and the measured variable is qualitative nominal: the type of solution in the Pareto sense (two modalities possible: Pareto-optimal solution and Dominated solution).

The contingency table is presented in Table 5 and describes the samples and calculated frequencies. 
Abi Akle A., Yannou B., minel S. (2019). Information visualization for efficient knowledge discovery and informed decision in Design by Shopping. Journal of Engineering Design, 30 (6), 227-253, doi: 10.1080/09544828.2019.1623383.

Table 5. Contingency table for the type of selected solution between the three groups

\begin{tabular}{|l|l|l|l|}
\hline & $\begin{array}{l}\text { Pareto-optimal } \\
\text { solution }\end{array}$ & Dominated solution & Total \\
\hline SPM (frequency) & $23(24.7)$ & $3(1.3)$ & 26 \\
\hline PCP (frequency) & $26(24.7)$ & $0(1.3)$ & 26 \\
\hline SSP (frequency) & $25(24.7)$ & $1(1.3)$ & 26 \\
\hline Total (frequency) & $74(0.95)$ & $4(0.05)$ & $78(1)$ \\
\hline
\end{tabular}

We found (from the descriptive statistics analysis) that over $20 \%$ of the values in the contingency table (half in our case) had an expected frequency of less than 5\% (i.e. 95\% for Pareto-optimal solution); we therefore applied the chi-squared test with the Yates correction to address our statistical assumptions: $X^{2}=1.647$, df $=2$ and $p=0.44$. We could infer that there was no difference between the three groups.

The first part of the analysis led us to conclude that there was no difference in knowledge between the three groups, and that they almost all selected a Pareto solution. We could thus divide up the groups for the subsequent analyses.

\section{Measurements during test: discovery variables}

Unfortunately, we had different sample sizes, because not all the subjects were able to perform all the tests owing to platform malfunctions. All the discovery variables measured were quantitative. We therefore applied the ANOVA-between statistical test and pairwise $t$-test post hoc analysis if necessary (i.e. if ANOVA was significant). We considered the following statistical hypotheses: H0: there is no difference between the graphs for the discovery phase and $\mathrm{H} 1$ : there is a difference.

For the Vessel problem, we obtained for the SSP $(n=12)$ an average of 2.66 discoveries, 209.6 seconds elapsed before the first discovery, and 198.4 seconds mean time per discovery, for PCP $(n=12) 3.25$ discoveries, 115.8 seconds elapsed before the first discovery, and 131.1 seconds mean time per discovery, and for SPM $(n=13) 6.3$ discoveries, 14.8 seconds elapsed before the first discovery, and 65.1 seconds mean time per discovery. ANOVAs give significant results: $\mathrm{F}(2,34)=24.27$ and $p<0.0001$ for the number of discoveries, $\mathrm{F}(2,33)=8.65$ and $p=0.000954$ for the time before the first discovery, and $\mathrm{F}(2,33)=6.45$ and $p=0.004321$ for the mean time per discovery (see Table 6).

For the Disk problem, we obtained for the SSP $(n=13)$ an average of 2.92 discoveries, 175.2 seconds elapsed before the first discovery, and 129.1 seconds mean time per discovery, for PCP $(n=14) 3$ discoveries, 107.2 seconds elapsed before the first discovery, and 93.3 seconds mean time per discovery, and for SPM $(n=13) 5.4$ discoveries, 52.4 seconds elapsed before the first discovery, and 75.3 seconds mean time per discovery. ANOVAs give significant results: $\mathrm{F}(2,37)=17.01$ and $p<0.0001$ for the number of discoveries, $\mathrm{F}(2,37)=9.17$ and $p=0.000583$ for the time before the first discovery and $\mathrm{F}(2,37)=5.71$ and $p=0.006899$ for the mean time per discovery (see Table $6)$. 
Abi Akle A., Yannou B., minel S. (2019). Information visualization for efficient knowledge discovery and informed decision in Design by Shopping. Journal of Engineering Design, 30 (6), 227-253, doi: 10.1080/09544828.2019.1623383.

Table 6. Results of ANOVA performed for the three discovery variables for the two problems

\begin{tabular}{|c|c|c|c|}
\hline Problem & $\begin{array}{l}\text { Number } \\
\text { discoveries }\end{array}$ & $\begin{array}{l}\text { Time before the first } \\
\text { discovery (seconds) }\end{array}$ & $\begin{array}{l}\text { Mean time to } \\
\text { complete a } \\
\text { discovery (seconds) }\end{array}$ \\
\hline \multirow[t]{2}{*}{ Vessel } & $F(2,34)=24.27$ & $F(2,33)=8.65$ & $F(2,33)=6.45$ \\
\hline & $p<0.0001$ & $p=0.000954$ & $p=0.004321$ \\
\hline \multirow[t]{2}{*}{ Disk } & $F(2,37)=17.01$ & $F(2,37)=9.17$ & $F(2,37)=5.71$ \\
\hline & $p<0.0001$ & $p=0.000583$ & $p=0.006899$ \\
\hline
\end{tabular}

All ANOVA p-values were less than $10 \%$, so the results were significant: there was a significant difference between the three groups (i.e. three graphs) for the three discovery variables and for solving both problems. We performed a post hoc analysis for the two problems with $t$-test pairwise comparison (see Table 7). The results of the t-tests are given in Table 7 and the averages for each variable are given in Figure 12.

Table 7. Results of the t-test for the three discovery variables for the two problems

\begin{tabular}{|c|c|c|c|c|}
\hline Problem & $\begin{array}{l}t \text {-test } \\
\text { pairwise } \\
\text { comparison }\end{array}$ & $\begin{array}{l}\text { Number } \\
\text { discoveries }\end{array}$ & $\begin{array}{l}\text { Time before the } \\
\text { first discovery }\end{array}$ & $\begin{array}{lr}\text { Mean time } & \text { to } \\
\text { complete } & \text { a } \\
\text { discovery } & \end{array}$ \\
\hline \multirow[t]{3}{*}{ Vessel } & SSP vs. PCP & $\begin{array}{l}t(22)=-0.92 \\
p=0.18\end{array}$ & $\begin{array}{l}t(21)=-1.57 p= \\
0.066\end{array}$ & $\begin{array}{l}t(21)=1.44 p= \\
0.0823\end{array}$ \\
\hline & SSP vs. SPM & $\begin{array}{l}t(23)=6.62 \\
p<0.0001\end{array}$ & $\begin{array}{l}t(22)=3.69 p= \\
0.0006\end{array}$ & $\begin{array}{l}t(22)=3.19 p= \\
0.002\end{array}$ \\
\hline & $\mathrm{PCP}$ vs. SPM & $\begin{array}{l}t(22)=5.86 \\
p<0.0001\end{array}$ & $\begin{array}{l}t(23)= \\
p<0.0001\end{array}$ & $\begin{array}{l}t(23)=3.52 p= \\
0.0009\end{array}$ \\
\hline \multirow[t]{3}{*}{ Disk } & SSP vs. PCP & $\begin{array}{l}t(25)=0.16 \quad p= \\
0.437\end{array}$ & $\begin{array}{l}t(25)=2.39 p= \\
0.0123\end{array}$ & $\begin{array}{l}t(25)=1.94 p= \\
0.0319\end{array}$ \\
\hline & SSP vs. SPM & $\begin{array}{l}t(24)=4.99 \quad p \\
<.0001\end{array}$ & $\begin{array}{lll}t(24)= & 4.44 & p \\
<.0001 & & \\
\end{array}$ & $\begin{array}{l}t(24)=3.24 p= \\
0.0017\end{array}$ \\
\hline & PCP vs. SPM & $\begin{array}{lll}t(25)= & 5.08 \quad p \\
<.0001 & & \end{array}$ & $\begin{array}{l}t(25)=1.89 p= \\
0.0352\end{array}$ & $\begin{array}{l}t(25)=1.44 p= \\
0.081\end{array}$ \\
\hline
\end{tabular}

The results of the $t$-test pairwise comparison indicate for the number of discoveries that there was a significant difference between SPM and SSP with $t=6.62$ and $p$-value less than 0.0001 for Vessel problem and $t=4.99$ and $p$-value less than 0.0001 for Disk problem, and SPM and PCP with $\mathrm{t}=5.86$ and $\mathrm{p}$-value less than 0.0001 for Vessel problem and $t=5.08$ and $p$-value less than 0.0001 for Disk problem. The results of the t-tests between the SSP and the PCP, for this discovery variable, do not allow us to conclude that there was a difference between them. The group that tested the SPM performed better than those that tested the SSP and PCP for the number of discoveries realized: They had made a greater number of discoveries in a statistical significant way.

Regarding the two other discovery variables, the t-test reveals that there was a significant difference for both problems between SSP, PCP and SPM. Indeed, all p-values resulting 
Abi Akle A., Yannou B., minel S. (2019). Information visualization for efficient knowledge discovery and informed decision in Design by Shopping. Journal of Engineering Design, 30 (6), 227-253, doi: 10.1080/09544828.2019.1623383.

from the t-tests are less than 10\% (see Table 7). Thereby SPM was significantly different from the other two and had the best scores (see Figure 12).

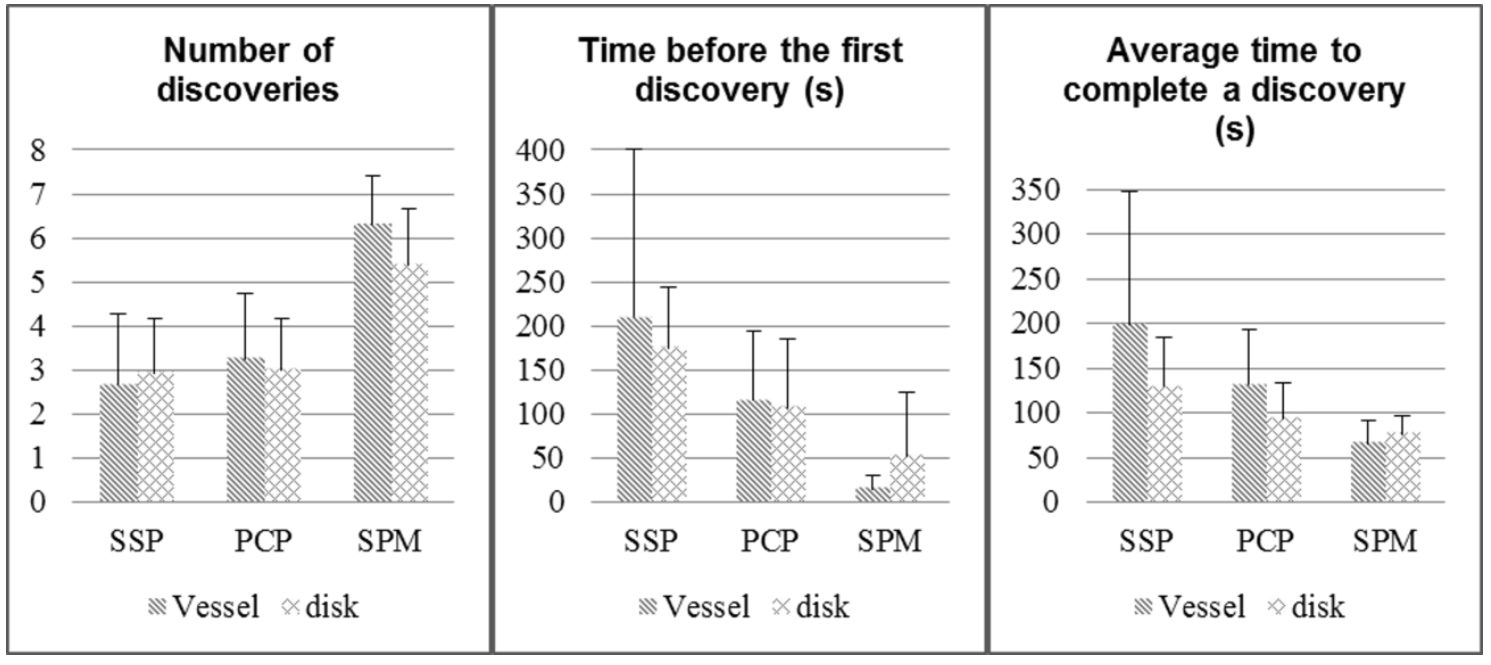

Figure 12. Average and standard deviation for the three discovery variables

Statistical analysis performed on three discovery variables led us to conclude that the scatter plot matrix (SPM) was the most relevant graph for the discovery phase. We note also that the simple scatter plot (SSP) was the graph that yielded the worst results for the discovery phase.

\section{Justification of the decision}

For this indicator we analysed the data from the two problems together. Our study had two qualitative variables: the group (SPM, PCP and SSP) and the answer to the question (decision justified or not). We applied a chi-squared test: $X^{2}=4.747, \mathrm{df}=2$ and $p=0.093$. The result was significant. We then ran the chi-squared tests in pairs:

- SSP vs. PCP: $X^{2}=0.18, \mathrm{df}=1$ and $p=0.671$

- SSP vs. SPM: $X^{2}=2.99, \mathrm{df}=1$ and $p=0.084$

- PCP vs. SPM: $X^{2}=4.447, \mathrm{df}=1$ and $p=0.035$

There was a significant difference between the SPM and SSP graphs, and SPM and PCP. Decisions were justified with SPM. We conclude that SPM was the graph with which the participants made the most informed decisions.

\section{Discussion}

Although we measured the participants' evolution of knowledge in exploration and visualization of the design space, we did the experimentation with students and therefore novice designers. However, we know that visualization and more broadly the interface is not just a flat layer between the user and computer, but is rather a complex, mediating, cultural artefact (Eikenes and Morrison 2010). Thus, it is linked to the user (ie. Designer). In fact, there are procedural differences in how novices and experts use multidimensional data visualization and exploration tools when solving an engineering design problem (Wolf et al. 2009). More generally, we know that mental representations of a design problem are different between experts and novices and they have different 
Abi Akle A., Yannou B., minel S. (2019). Information visualization for efficient knowledge discovery and informed decision in Design by Shopping. Journal of Engineering Design, 30 (6), 227-253, doi: 10.1080/09544828.2019.1623383.

behaviours in how they approach design tasks (Ahmed, Wallace and Blessing 2003). Experts reveal location of more interconnections (Björklund 2013). This ability could modify the results obtained because the interconnections are discoveries. So, distinction between expert and novice profile should improve results.

Also, the graphs tested in our experiment have limits related to the habits of the designers. The training will change the performance related to the use of the PCP. On the one hand, it is seen in (Abi Akle et al., 2017) that only 3.3\% of their testers use PCP and on the other hand, Wolf et al. (2011) show the importance of training for the entire process of exploring the trade space.

The experiment was conducted on a parametric sizing activity. However, Design by Shopping extends to the conceptual and detailed phase of the design process. It would be wise to test graphs / representations of information for the selection of concepts or technological choices in order to identify those that would make it possible to be effective and to facilitate the gain of knowledge for an informed decision in these situations. For example, Mattson and Messac (2005) propose a multiple Pareto front (s-Pareto frontier) grouping together feasible solutions of different generated concepts. In this work, the authors focus on the benefit of optimization algorithms for design selection considering uncertainty. In this sense, our experimental protocol should be applied to solving more complex design problems: more or less defined problems with uncertainty or problems composed of several subsystems.

Our experiment allowed to test a design situation in which the designers manipulate a large design space with an infinity of possible solutions. In the case of a limited number of solutions, the efficient graphs could be different from those tested in our works. Indeed, when selecting suitable graphs according to Miettinen's work (2014), we eliminated those that do not allow the visualization of a very large number of design points. The efficiency of a graph for a large number of solutions may be inefficient for a smaller number of solutions, for example the "circle segment" which is useful for data sets comprising a colossal number (i.e. two hundred thousand to two million) because with this graph a data corresponds to a pixel. Indeed, if the set to be visualized is composed of a thousand data, the visual would be reduced or poor (Keim, 2000). In addition, our work focused on the visualization of the design points that populate the design space. Others, graphs allow the visualization of the design space in a continuous way like the tool VIDEO of Kollat and Reed (2007). Testing these graphs and comparing them to those tested in our work would improve our results.

Finally, in our experimental protocol, we made the choice to identify the discoveries made by the participants by analysing the video and eye-tracking recording. The use of the Think aloud method combined with video and eye-tracking recording would have made it possible to refine the results. The Think aloud method is very useful for obtaining qualitative data in real time but has a bias that the participant "thinking aloud" questions what s/he says and does. This situation changes the behavior of the tester when s/he is not expert on the task performed. We performed experiments with novice designers, so we opted not to use this method. Reproducing our protocol with expert designers would allow the addition of Think aloud and thus improve the results.

\section{Conclusion}

We present an optimization strategy to focus on informed decision as an instance of competiveness leverage. Designers have to build a design iteratively based on 
Abi Akle A., Yannou B., minel S. (2019). Information visualization for efficient knowledge discovery and informed decision in Design by Shopping. Journal of Engineering Design, 30 (6), 227-253, doi: 10.1080/09544828.2019.1623383.

experimentation. They have to choose from a large, often cumbersome set of alternatives. This work helps gain a better understanding of how data presentation impacts on understanding of the design problem, on the design decision-making and thus on system performance. We identify the simple scatter plot (SSP), the scatter plot matrix (SPM) and the parallel coordinate plot (PCP) as useful graphs for Design Space Exploration.

Our findings enable us to draw out clear recommendations for the choice of a graph for the discovery phase in design space exploration to make an informed decision. We have shown how to select an optimal solution from among a set of feasible solutions defined by their design and performance value vectors. The results of our tests show that design space exploration was improved when using SPM graph to present data. Designers seemed more confident and made informed decisions depending on the graphical interface proposed. SPM is most appropriate for the discovery phase, because this phase involves understanding the problem by observing interactions between the variables, and SPM is the graph that best reveals these types of interactions (such as clusters, correlations, etc.) (Keim 2000). Moreover, we believe that it can help designers innovate because it uses Cartesian representations (i.e. facilitates interpretation), and gives an overview of the dataset, and therefore of the problem. Our results bring a benefit to the engineering designers allowing them to explore the design space in better conditions. Our findings give a better apprehension of the Design Space Exploration method which is a paradigm of Design by Shopping. Indeed, our work reinforces the use of the method by clarifying the efficiency of the tools available to the engineers. For the future, we will continue work on our model to refine and adapt it to different archetypes of designers and engineers' expertise.

Conflict of Interest: The authors report no conflicts of interest.

\section{References}

Abi Akle, A., S. Minel, and B. Yannou. 2015. "Graphical support adapted to designers for the selection of an optimal solution in design by shopping." In DS 80-6 Proceedings of the 20th International Conference on Engineering Design (ICED 15) Vol 6: Design Methods and Tools-Part 2 Milan, Italy, 27-30.07. 15.

Abi Akle, A., S. Minel, and B. Yannou. 2017. "Information visualization for selection in Design by Shopping." Research in Engineering Design 28(1): 99-117. DOI: 10.1007/s00163-016-0235-2.

Ahmed, S., Wallace, K. M., \& Blessing, L. T. 2003. "Understanding the differences between how novice and experienced designers approach design tasks". Research in engineering design, 14(1), 1-11.

Andrienko, G., and N. Andrienko. 2001. "Constructing parallel coordinates plot for problem solving." In 1st International Symposium on Smart Graphics (pp. 9-14).

Balling, R. 1999. "Design by shopping: A new paradigm?." In Proceedings of the Third World Congress of structural and multidisciplinary optimization (WCSMO-3) (Vol. 1, pp. 295-297).

Barron, K., T. W. Simpson, L. Rothrock, M. Frecker, R. R. Barton, and C. Ligetti. 2004. "Graphical user interfaces for engineering design: impact of response delay and training on user performance." In ASME 2004 International Design Engineering Technical Conferences and Computers and Information in Engineering Conference (pp. 11-20). American Society of Mechanical Engineers. 
Abi Akle A., Yannou B., minel S. (2019). Information visualization for efficient knowledge discovery and informed decision in Design by Shopping. Journal of Engineering Design, 30 (6), 227-253, doi: 10.1080/09544828.2019.1623383.

Bass, T. 2000. "Intrusion detection systems and multisensor data fusion." Communications of the ACM 43(4): 99-105. DOI: 10.1145/332051.332079.

Björklund, T. A. 2013. "Initial mental representations of design problems: Differences between experts and novices". Design Studies, 34(2), 135-160.

Canbaz, B. (2013). "Preventing and resolving design conflicts for a collaborative convergence in distributed set-based design." PhD diss., Ecole Centrale Paris.

Chandrasegaran, S. K., K. Ramani, R. D. Sriram, I. Horváth, A. Bernard, R. F. Harik, and W. Gao. 2013. "The evolution, challenges, and future of knowledge representation in product design systems." Computer-Aided Design 45(2): 204228. DOI: $10.1016 /$ j.cad.2012.08.006.

Daskilewicz, M. J., and B. J. German, B. 2012." Rave: A computational framework to facilitate research in design decision support." Journal of computing and information science in engineering 12(2): 021005. DOI: 10.1115/1.4006464.

Deb, K., and A. Srinivasan. 2006." Innovization: Innovating design principles through optimization." In Proceedings of the 8th annual conference on Genetic and evolutionary computation (pp. 1629-1636). ACM.

Eikenes JOH, Morrison A 2010. "Navimation: exploring time, space \& motion in the design of screen-based interfaces". Int J Des 4(1):1-16

Gettinger, J., E. Kiesling, C. Stummer, and R. Vetschera. 2013. "A comparison of representations for discrete multi-criteria decision problems." Decision support systems 54(2): 976-985. DOI: 10.1016/j.dss.2012.10.023.

Glaser, S. D., and A. Tolman. 2008. "Sense of sensing: from data to informed decisions for the built environment." Journal of infrastructure systems 14(1): 4-14. DOI: 10.1061/(ASCE)1076-0342(2008)14:1(4).

Inselberg, A. 2009. "Parallel Coordinates: VISUAL Multidimensional Geometry and its Applications."

Ireson, N. 2009. "Local community situational awareness during an emergency." In Digital Ecosystems and Technologies, 2009. DEST'09. 3rd IEEE International Conference on (pp. 49-54). IEEE.

Kanji, Gopal K. 2006. 100 Statistical Tests. Sage Publications.

Keim, D. A. 2000. "Designing pixel-oriented visualization techniques: Theory and applications." IEEE Transactions on Visualization and Computer Graphics 6(1): 59-78.

Keim, D. A., F. Mansmann, J. Schneidewind, and H. Ziegler. 2006. "Challenges in visual data analysis." In Information Visualization, 2006. IV 2006. Tenth International Conference on (pp. 9-16). IEEE.

Keim, D., G. Andrienko, J. D. Fekete, C. Görg, J. Kohlhammer, and G. Melançon. 2008a. Visual analytics: Definition, process, and challenges. Lecture notes in computer science, 2008, vol. 4950, p. 154-176. Springer Berlin Heidelberg.

Keim, D. A., F. Mansmann, J. Schneidewind, J. Thomas, and H. Ziegler. 2008b. "Visual analytics: Scope and challenges." In Visual data mining (pp. 76-90). Springer Berlin Heidelberg.

Kollat, J. B., and P. Reed. 2007. "A framework for visually interactive decision-making and design using evolutionary multi-objective optimization (VIDEO)." Environmental Modelling \& Software 22(12): 1691-1704. DOI: 10.1016/j.envsoft.2007.02.001.

Ligetti, C., T. W. Simpson, M. Frecker, R. R. Barton, and G. Stump. 2003. "Assessing the impact of graphical design interfaces on design efficiency and effectiveness." 
Abi Akle A., Yannou B., minel S. (2019). Information visualization for efficient knowledge discovery and informed decision in Design by Shopping. Journal of Engineering Design, 30 (6), 227-253, doi: 10.1080/09544828.2019.1623383.

Journal of Computing and Information Science in Engineering 3(2): 144-154. DOI: $10.1115 / 1.1583757$.

Lurie, N. H., and C. H. Mason. 2007. "Visual representation: Implications for decision making." Journal of Marketing 71(1): 160-177. DOI: 10.1509/jmkg.71.1.160.

Mattson, C. A., \& Messac, A. 2005. "Pareto frontier based concept selection under uncertainty, with visualization". Optimization and Engineering, 6(1), 85-115.

Mavris, D. N., O. J. Pinon, and D. Fullmer Jr. 2010. "Systems design and modeling: A visual analytics approach." In Proceedings of the 27th International Congress of the Aeronautical Sciences (ICAS), Nice, France.

Meyer, J., J. Thomas, S. Diehl, B. D. Fisher, D. A. Keim, D. Laidlaw, and A. Ynnerman. 2010. "From Visualization to Visually Enabled Reasoning." Scientific visualization: Advanced concepts 1: 227-245. $\quad$ DOI: 10.4230/DFU.SciViz.2010.227

Miettinen, K. (2014). Survey of methods to visualize alternatives in multiple criteria decision making problems. OR Spectrum 36(1), 3-37. DOI: 10.1007/s00291-0120297-0.

Miller, S. W., T. W. Simpson, M. A. Yukish, L. A. Bennett, S. E. Lego, and G. M. Stump. 2013. "Preference Construction, Sequential Decision Making, and Trade Space Exploration.” In ASME 2013 International Design Engineering Technical Conferences and Computers and Information in Engineering Conference (pp. V03AT03A014-V03AT03A014). American Society of Mechanical Engineers.

Otto, K. N., and E. K. Antonsson. 1993. "The method of imprecision compared to utility theory for design selection problems." Design Theory and MethodologyDTM'93, 167-173.

Pahl, G., and Beitz, W. 2013. Engineering design: a systematic approach. Springer Science \& Business Media.

Petersen, S., and S. Svendsen. 2010. "Method and simulation program informed decisions in the early stages of building design." Energy and buildings 42(7): 1113-1119. DOI: 10.1016/j.enbuild.2010.02.002.

Riveiro, M., G. Falkman, and T. Ziemke. 2008. "Visual analytics for the detection of anomalous maritime behavior." In Information Visualisation, 2008. IV'08. 12th International Conference (pp. 273-279). IEEE.

Russell, A. D., C. Y. Chiu, and T. Korde. 2009. "Visual representation of construction management data." Automation in Construction 18(8): 1045-1062. DOI: 10.1016/j.autcon.2009.05.006.

Simpson, T. W., M. Frecker, R. R. Barton, and L. Rothrock. 2007. "Graphical and textbased design interfaces for parameter design of an I-beam, desk lamp, aircraft wing, and job shop manufacturing system." Engineering with Computers 23(2): 93-107. DOI: 10.1007/s00366-006-0045-7.

Simpson, T. W., D. B. Spencer, M. A. Yukish, and G. Stump. 2008. "Visual steering commands and test problems to support research in trade space exploration." In 12th AIAA/ISSMO Multidisciplinary Analysis and Optimization Conference (pp. 10-12).

Siirtola, H. 2007. "Interactive visualization of multidimensional data." Tampereen yliopisto.

Stump, G., Simpson, T. W., Donndelinger, J. A., Lego, S., \& Yukish, M. 2009. “Visual steering commands for trade space exploration: User-guided sampling with example". Journal of Computing and Information Science in Engineering, 9(4), 044501-1. 
Abi Akle A., Yannou B., minel S. (2019). Information visualization for efficient knowledge discovery and informed decision in Design by Shopping. Journal of Engineering Design, 30 (6), 227-253, doi: 10.1080/09544828.2019.1623383.

Stump, G., M. Yukish, J. D. Martin, and T. W. Simpson. 2004. "The ARL trade space visualizer: An engineering decision-making tool." In 10th AIAA/ISSMO Multidisciplinary Analysis and Optimization Conference (Vol. 30).

Sullivan, K. J., W. G. Griswold, Y. Cai, and B. Hallen. 2001. "The structure and value of modularity in software design.” In ACM SIGSOFT Software Engineering Notes (Vol. 26, No. 5, pp. 99-108). ACM.

Ware, C. 2004. Information Visualization: Perception for Design (Interactive Technologies). Morgan Kaufmann 2nd edition.

Wegman, E. J. 1990. "Hyperdimensional data analysis using parallel coordinates." Journal of the American Statistical Association 85(411): 664-675.

Wiederhold, G. 1992. "Mediators in the architecture of future information systems." Computer, 25(3): 38-49. DOI: 10.1109/2.121508.

Wolf D, Simpson TW, Zhang XL 2009. "A preliminary study of novice and expert users' decision-making procedures during visual trade space exploration". In: ASME 2009 international design engineering technical conferences and computers and information in engineering conference, paper no. DETC200987294, San Diego, California, USA, pp 1361-1371

Wolf D, Hyland J, Simpson TW, Zhang XL 2011. "The importance of training for interactive trade space exploration: a study of novice and expert users". J Comput Inf Sci Eng 11(3):031009

Wood, K. L., K. N. Otto, and E. K. Antonsson. 1992. "Engineering design calculations with fuzzy parameters.” Fuzzy Sets and Systems 52(1): 1-20. DOI: 10.1016/01650114(92)90031-X.

Yan, X., M. Qiao, J. Li, T. W. Simpson, G. M. Stump, and X. Zhang. 2012. "A WorkCentered Visual Analytics Model to Support Engineering Design with Interactive Visualization and Data-Mining.” In System Science (HICSS), 2012 45th Hawaii International Conference on (pp. 1845-1854). IEEE.

Yannou, B., F. Moreno, H. J. Thevenot, and T. W. Simpson. 2005. "Faster generation of feasible design points." In ASME 2005 International Design Engineering Technical Conferences and Computers and Information in Engineering Conference (pp. 355-363). American Society of Mechanical Engineers.

Zhang, X., Simpson, T., Frecker, M., \& Lesieutre, G. 2012. "Supporting knowledge exploration and discovery in multi-dimensional data with interactive multiscale visualization". Journal of Engineering Design, 23(1), 23-47. 
Abi Akle A., Yannou B., minel S. (2019). Information visualization for efficient knowledge discovery and informed decision in Design by Shopping. Journal of Engineering Design, 30 (6), 227-253, doi: 10.1080/09544828.2019.1623383.

\section{Appendices}

\section{Multiple-choice questionnaire MCQ\#1 for Scatter Plot Matrix (SPM) group}

1. What is a design point?
a) A solution possible
b) A step in the design process
c) A sub-system to design
d) I do not know

2. What is a Pareto-optimal solution?
a) The best possible solution
b) A non-dominated solution
c) A solution that respects the law of $80 / 20$
d) I do not know

3. How to find a robust solution? (or what is a robust solution?)
a) Analyze the lifetime of a solution
b) Analyze the sensitivity of performance variables
c) Analyze the variability of input on the response variable
d) I do not know

4. What is the goal of the design space exploration?
a) Search for optimal solutions in a design space
b) Search Pareto front in a design space
c) Search for standards in a design space
d) I do not know

5. Why to use a « highlight » function?
a) To find the optimal solutions
b) To visualize an additional variable
c) To isolate some design points
d) I do not know

6. What is the purpose of the «brush » function?

a) Smoothing points that are not optimal 
Abi Akle A., Yannou B., minel S. (2019). Information visualization for efficient knowledge discovery and informed decision in Design by Shopping. Journal of Engineering Design, 30 (6), 227-253, doi: 10.1080/09544828.2019.1623383.
b) Display the detail of a point
c) Isolate points to highlight them
d) I do not know

7. What should I do if I want to see only the non-dominated Pareto points?
a) Use the "sampler" of non-dominated
b) Use sliders in the preference controller
c) Use the "highlight" button in the preference controller
d) I do not know

(6)

8. What is the interaction between variables $\mathrm{A}$ and $\mathrm{B}$ ?
a) Clusters
b) 2 transition points
c) A correlation
d) I do not know

9. Looking at the graph, which set of

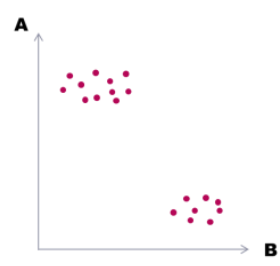
robust?
a) Blue
b) Purple
c) Red
d) I do not know

10. If I want to maximize

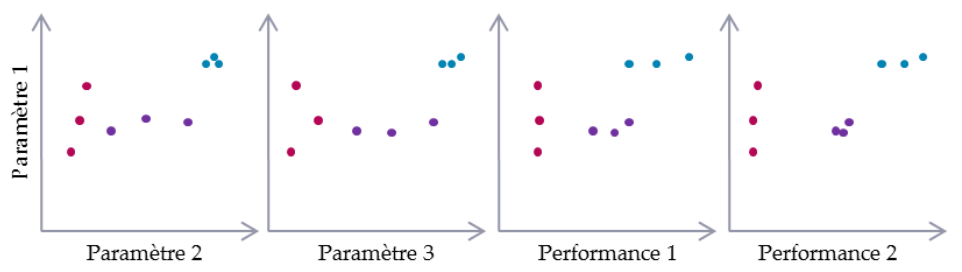
the variables s1 and s2 which picture corresponds?

a)

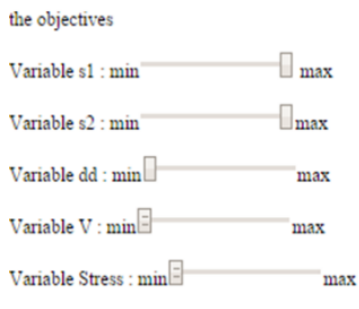

showle b)

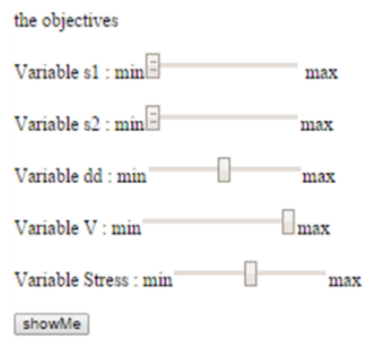

c)

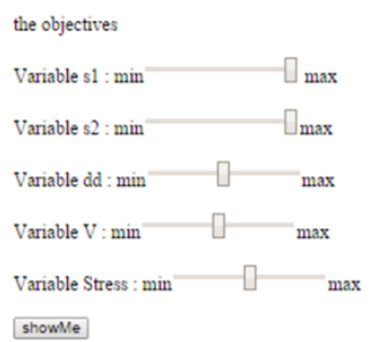

